\title{
Estimation of signal coherence threshold and concealed spectral lines applied to detection of turbofan engine combustion noise
}

\author{
Jeffrey Hilton Miles ${ }^{\text {a) }}$ \\ NASA John H. Glenn Research Center at Lewis Field, Cleveland, Ohio 44135
}

(Received 23 May 2010; revised 24 December 2010; accepted 30 December 2010)

\begin{abstract}
Combustion noise from turbofan engines has become important, as the noise from sources like the fan and jet are reduced. An aligned and un-aligned coherence technique has been developed to determine a threshold level for the coherence and thereby help to separate the coherent combustion noise source from other noise sources measured with far-field microphones. This method is compared with a statistics based coherence threshold estimation method. In addition, the un-aligned coherence procedure at the same time also reveals periodicities, spectral lines, and undamped sinusoids hidden by broadband turbofan engine noise. In calculating the coherence threshold using a statistical method, one may use either the number of independent records or a larger number corresponding to the number of overlapped records used to create the average. Using data from a turbofan engine and a simulation this paper shows that applying the Fisher z-transform to the un-aligned coherence can aid in making the proper selection of samples and produce a reasonable statistics based coherence threshold. Examples are presented showing that the underlying tonal and coherent broad band structure which is buried under random broadband noise and jet noise can be determined. The method also shows the possible presence of indirect combustion noise. [DOI: 10.1121/1.3546097]
\end{abstract}

PACS number(s): 43.60.Ac, 43.60.Cg, 43.28.Kt [EJS]

Pages: 3068-3081

\section{NOMENCLATURE}

$B_{e} \quad$ Resolution bandwidth, $\mathrm{Hz}, B_{e}=\Delta_{f}=1 / T_{d}$ $=r / N P=11.71875 \mathrm{~Hz}$

$D$ Number of points contained in deliberate time delay shift interval, $D=r \tau_{1}$

$E_{I} \quad$ Confidence interval

$f$ Frequency, $\mathrm{Hz}$

$\hat{G}_{x x}(f)$ Auto power spectral density function defined for non-negative frequencies only (one sided)

$\hat{G}_{x y}(f)$ Cross power spectral density function defined for non-negative frequencies only (one sided)

$L \quad$ Number of degrees of freedom

$L_{y} \quad$ Number of frequencies, $f_{c} / \Delta f=N P / 2$ (2048)

$n$ Number of frequencies used in simulation

N1 Corr. Corrected rotor speed, rpm

$n_{1}, n_{2}$, and $n_{3}$ Independent stationary Gaussian random time series

$n_{d} \quad$ Number of disjoint (independent) segments used in spectra estimates, $n_{d}=B_{e} T_{\text {total }}=234$

$n_{s} \quad$ Number of segments/blocks with overlap

$N P \quad$ Segment length, number of data points per segment (4096)

$P_{I} \quad$ Probability confidence interval, \%

$r$ Sample rate, samples/s (48 000)

$t$ Time, $\mathrm{s}$

$T_{\text {total }} \quad$ Total record length, $\mathrm{s}(\approx 20 \mathrm{~s})$

$T_{d}(i)$ Record length of data segment $i, T_{\text {total }} / n_{d}$, $\frac{N P}{r}, T_{d}=\frac{N P}{r}=4096 / 48000=0.08533 \mathrm{~s}$

\footnotetext{
${ }^{a)}$ Author to whom correspondence should be addressed. Electronic mail: Jeffrey.H.Miles@nasa.gov
}

$$
\begin{array}{rl}
\hat{W}_{s_{F} s_{F}} & \text { The coherent output power function } \\
w & w \text { transform function, } w\left(\hat{\gamma}_{x y}\right)=\tanh ^{-1}\left(\hat{\gamma}_{x y}\right) \\
x(t) & \text { Signal } x \text { time history } \\
x_{n}(t), y_{n}(t) & \text { Noise time series } \\
\text { and } z_{n}(t) & \\
y(t) & \text { Signal } y \text { time history } \\
z & z \text { transform function, } z(x)=\tanh ^{-1}(\sqrt{x}) \\
x & \text { Signal } x \\
y & \text { Signal } y \\
\alpha & \text { Noise time series mixing parameter } \\
\Delta f & \text { Frequency step, } 1 / T_{d}, \mathrm{~Hz}(11.718) \\
\Delta t & \text { Sampling interval, } 1 / r=1 / 48000), \mathrm{s} \\
\hat{\gamma}_{x_{n} y_{n}}^{2}\left(f, P_{I}, n\right) & \text { Coherence threshold using a probability, } P_{I}, \\
& \text { and } n \text { records, } n_{d} \\
\hat{\gamma}_{x_{n} y_{n}}^{2}(n) & \text { Coherence threshold using the number of } \\
& \text { records, } n \text {, and a 95\% confidence interval } \\
\hat{\gamma}_{x y}^{2}(f) & \text { Estimated magnitude squared coherence } \\
& \text { (MSC) function } \\
\left|\hat{\gamma}_{x_{n} y_{n}}^{2}(f)\right| & \text { Estimated un-aligned MSC function of } \\
\left\{\hat{\gamma}_{x_{n} y_{n}}^{2}(f)\right\}^{T} & \text { signals } \\
\tau_{1} \quad \text { Simulated coherence target value } \\
\text { Deliberate time delay shift interval, } \tau_{1}=D / r, \mathrm{~s}
\end{array}
$$

\section{INTRODUCTION}

Identification of noise sources in the core of a turbofan engine is difficult since the core noise signal consists of broadband noise, tonal noise, and hydrodynamic pressure perturbations. This paper discusses measurements made by external far-field microphones and pressure sensors in the combustor. All the spectra and cross-spectra are estimated using Welch's non-parametric method which is based on averaging multiple windowed periodograms using overlapping time sequences. ${ }^{1}$ The method is discussed in textbooks by Canday, ${ }^{2}$ Stearns and David, ${ }^{3}$ and by Manolakis et l. $^{4}$ 
Using these spectra and cross-spectra, the magnitude squared coherence (MSC) between a combustor sensor and a far-field microphone is calculated to measure the similarity of the amplitude variations at particular frequencies. Interpreting the coherence measurement was made difficult due to the presence of an external extraneous low frequency noise source, as discussed by Miles ${ }^{5}$ that reduces the measured coherence and required the calculation of a coherence threshold. The coherence threshold would be the coherence of two independent signals measured in the same manner which would indicate the lowest coherence measurable for two independent signals for a particular system. If the measured coherence is below the threshold, it is attributed to random noise. This paper presents the method used to establish the coherence threshold used for the turbofan engine data. Then results are presented, which show that the measured coherence between the combustor and the signal at a far-field microphone while small is above the coherence threshold.

A statistical test for independence is discussed by Carter ${ }^{6,7}$ Halliday et al., ${ }^{8}$ and Brillinger. ${ }^{9}$ It provides an equation to calculate the upper $95 \%$ confidence limit for the case when the measured functions are independent. The statistical test is a confidence interval, which is function of the number of independent samples (record segments), $n_{d}$. The confidence interval is based on a statistical distribution of the magnitude squared coherence (MSC) estimates, $\hat{\gamma}_{x y}^{2}$, computed from signals produced by two Gaussian random processes that have been divided into $n_{d}$ independent segments having spectral densities estimated by Welch's averaged periodogram method without overlapping segments. The test does not take into account the procedure for calculating the coherence such as the amount of sample overlap and does not account non-Gaussian signals containing tones. The statistical test results are of interest because they provide a confidence interval independent of frequency.

This statistical test for independence produces a threshold value that does not depend on the measurement procedure used. However, selection of the signal processing calculation procedure is the responsibility of the researcher. Consequently, a coherence threshold value may depend on the selection of the non-parametric coherence signal processing calculation procedure such as the Blackman-Tukey method discussed by Blackman and Tukey ${ }^{10}$ and by Manolakis et al. ${ }^{4}$ or Welch's method discussed by Welch, ${ }^{1}$ and by Manolakis et l. $^{4}$ It is also a function of the selection of a spectral window function as discussed by Canday, ${ }^{2}$ Stearns and David, ${ }^{3}$ and Manolakis et al. ${ }^{4}$ Another variable is the selection of the amount of segment overlap. In addition, the coherence threshold might depend on the statistical distribution of the time series and the presence of tones also.

In an earlier paper, Miles ${ }^{11}$ discusses a procedure that uses Welch's method to calculate the cross-spectra of two time histories recorded simultaneously with the modification of deliberately translating one of them with respect to the other by a time delay interval, $\tau_{1}$, that exceeds the sample record length, $T_{d}$, of the averaging periodogram spectrum analyzer used. This procedure creates two distinct independent time histories that will have the same sinusoidal content. The measured coherence at frequencies where tones are not present will have a threshold value of two independent signals. In addition, it is calculated on the identical measurement system used in calculating the aligned signal coherence. Since the translation does not change the phase relationships in the time sequence, all tonal information will appear. The procedure at the same time as it calculates a coherence threshold also reveals periodicities, spectral lines, and undamped sinusoids hidden or concealed by broadband turbofan engine noise. This calculated coherence threshold will be designated as $\left|\hat{\gamma}_{x_{n} y_{n}}^{2}(f)\right|$. Moreover, the two time series processed can be identical. Then one produces a coherence of the auto-spectrum that shows the coherence threshold of the system and tones because all broadband random information is removed.

As a consequence, the results of the statistical test for independence can now be directly compared with an estimated coherence value produced by a simple signal analysis calculation procedure. A surrogate data analysis procedure to calculate only the threshold coherence was studied by Faes et al., ${ }^{12}$ and it is discussed in Sec. II. These measurement procedures endow the threshold coherence with a reality and may help identify subtle noise sources.

The total turbofan engine data record length studied herein is about $20 \mathrm{~s}$ and engine tones are persistent throughout this interval. The tones are of interest so that some of the irregularities in the auto-spectrum can be identified and tones below the spectrum can be observed. Tracking spectral lines in signals with additive noise as a function of time from discrete-time observations is a well-known problem sometimes called in the time series literature the identification of sinusoids-in-noise problem or the hidden periodicity problem. Currently, problem is generally treated using a modern approach such as parametric spectral-estimation. One of the first signal processing textbooks discussing the classical and more modern approaches is by Candy. ${ }^{2}$ A more recent signal processing textbook discussing the classical and more modern approaches is by Manolakis et al. ${ }^{4} \mathrm{~A}$ mathematical approach to classical and modern signal processing topics is presented in book by Byrne. ${ }^{13}$ The topic of sinusoids-innoise is mentioned by Byrne. ${ }^{13}$ Several approaches to solve the problem of sinusoids-in-noise are discussed in the book by Quinn and Hannan. ${ }^{14}$ This identification of sinusoids-innoise problem which has applications to radar, communications, audio, power system control, and medical fields is solved using very different methods than the one identified herein. The un-aligned coherence method is not designed for tone tracking.

As discussed by Miles, ${ }^{11}$ the un-aligned coherence method is related to the time delay bias error. It is wellknown that if two time series are misaligned by a time delay interval, $\tau_{1}$, the estimated MSC for this case, $\hat{\gamma}_{x y}^{2}$, is reduced by the square of 1 minus the ratio of the time delay, $\tau_{1}$ to the data segment record length, $T_{d}$

$$
\left|\hat{\gamma}_{x y}^{2}\right|=\frac{\left|\hat{G}_{x y}\right|^{2}}{\hat{G}_{x x} \hat{G}_{y y}}=\left(1-\frac{\left|\tau_{1}\right|}{T_{d}}\right)^{2}\left|\hat{\gamma}_{x y}^{2}\right| .
$$


This relationship has been used to explain time delay bias by Bendat and Piersol. ${ }^{15}$ The importance of eliminating time delay bias and realigning time series by displacing one of them with respect to the other to maximize coherence has been mentioned in books by Jenkins and Watts, ${ }^{16}$ Priestley, ${ }^{17}$ and Brillinger ${ }^{9}$ and in articles by Carter. ${ }^{7,18}$ The concept used herein involves using a deliberate time delay that exceeds the sample record length, for example, letting $\tau_{1} \approx 1.5 T_{d}$ to find the coherence threshold, hidden periodicities, concealed spectral lines, and undamped sinusoids in turbofan engine signals. None of the above references mention this deliberate unalignment procedure. The only mention of its use appears in paper by Gross et al. ${ }^{19}$ in the medical literature where it is used to determine a coherence threshold without any mention of the origin of the procedure and for a study where no mechanical tones are present.

In the previous paper by Miles, ${ }^{11}$ Welch's averaged periodogram calculation procedure using a $50 \%$ overlap, as advised by Carter et al., ${ }^{18}$ was used to calculate the turbofan engine coherence. The overlap reduces the variance and as a consequence the number of samples of the time history used to calculate the coherence was $n_{s}=2 n_{d}$ where $n_{d}$ is the number of independent samples or records. The statistical procedure used to calculate the threshold as derived is based on the number of independent samples, $n_{d}$. The threshold value decreases as $n_{d}$ increases. In the previous paper by Miles, ${ }^{11}$ a simulation was done, which indicated that for Gaussian distributed random variables, the statistical test for the coherence threshold could use twice the number of independent records for a 50\% overlap in signal processing rather than the number of independent records. Just as the choice of window function in a non-parametric spectral-estimation, calculation is a matter of judgment, so is the choice of $n_{s}$ in calculating the statistical coherence threshold. The present paper more closely examines this premise. It indicates how applying the Fisher z-transform ${ }^{20}$ of the un-aligned coherence can aid in making the proper selection of samples, i.e., $n_{s}$ and produce a reasonable statistics based coherence threshold. The present paper presents results of a computer simulation and a turbofan engine test program. The results show that for the turbofan engine data set using the number of independent records, $n_{s}=n_{d}$ produces a higher threshold which is more reasonable. However, the use of $n_{s}=2 n_{d}$ which produces a lower threshold is fine for the computer simulation results which used a Gaussian distribution of random numbers. Results are presented, which show that while the coherence is low it is still above the higher coherence threshold based on using $n_{s}=n_{d}$ and a small but discernable contribution of the total noise is due to combustion noise.

Internal noise sources (i.e., core noise) become the significant contributors to overall turbofan engine noise during takeoff or approach when the fan and jet noise are reduced because of the forward velocity effects. The core noise consists of noise associated with combustor, compressor, and turbine noise. This paper discusses an extension of the three signal coherence technique to study the compressor and turbine noises and an extension of the coherent output power technique to study the combustor noise. The three signal coherence technique was developed by Chung et al. ${ }^{21}$ and
Chung $^{22}$ for flow noise rejection. The basic formulation for the coherent output power spectrum is presented by Bendat and Piersol. ${ }^{15}$ These methods depend on the measurement of the MSC function, $\hat{\gamma}_{x y}^{2}$. The coherence values are low so that to interpret these measurements, it is necessary to know the coherence threshold.

The data analyzed is from a Pratt \& Whitney PW4098 turbofan engine (East Hartford, CT). The test was conducted as a part of the NASA Engine Validation of Noise Reduction Concepts (EVNRC) Program in 2001. Two pressure transducers are mounted in the combustor and four far-field microphones are used. The combustor pressure transducers failed slowly during the test. Consequently, a sensor validation analysis was conducted based on the aligned and unaligned coherence method and is reported by Miles. ${ }^{23}$ The PW4098 combustor pressure sensor cross-spectra amplitude and phase plots resembled the cross-spectra used in the acoustic modal analysis of an AVCO Lycoming YF102 combustor (Stratford, CT), installed in a ducted test rig. This YF102 test program was conducted by Karchmer. ${ }^{24}$ This suggested that the observed structure in the coherence was due to a modal pattern. Consequently, using a model of the physics of pressure waves propagating in annular ducts as discussed by Tyler and Sofrin ${ }^{25}$ in a treatment of axial flow compressor noise, a procedure discussed by $\mathrm{Miles}^{26}$ was developed that uses the available combustor pressure sensor data to obtain a descriptions of the combustion modes in the annular combustor. Results from this work were used to select the signals to be analyzed and to interpret the results in an attempt to identify combustion noise in the far-field data. Work was then done that showed that by comparing aligned coherent power spectra calculated using the estimated time delay with the un-aligned coherent power spectra calculated with no time delay one can identify combustion noise. In addition, it was shown that conventional statistical methods could be used to detect correlated combustion noise and create a one parameter measure of its presence or absence. It was shown that the correlated combustion noise is only a small part of the total noise and that the plane wave combustion mode is apparent at low engine speeds. The aligned and un-aligned coherence method used in this work is discussed herein. Results are illustrated with examples from studies made of the Pratt and Whitney P\&W 4098 turbofan engine data.

The aligned and un-aligned coherence procedure of Miles ${ }^{11}$ was used to develop a method to validate combustor coherence measurements by Miles ${ }^{23}$ and to find the coherence threshold in a study of turbofan engine combustor annular duct modes by Miles. ${ }^{26}$ The method was also used to find the coherence threshold in analyzing core noise measurements made in a smaller Honeywell TECH977 turbofan engine (Phoenix, AZ). The smaller engine test results are reported in papers by Miles discussing a direct and indirect combustion noise source separation research program. ${ }^{27-29}$ The core noise results discussed herein will be interpreted in terms of these more recent results.

The deliberate decorrelation of signals was used in a study of flow-induced sound of wall-mounted cylinders with different geometries by Becker et al., ${ }^{30}$ a study of source 
separation from multiple microphone measurements in the far-field of a full scale aero engine by Mendoza et al. ${ }^{31}$ and in an engine validation of noise and emission reduction technology report by Weir. ${ }^{32}$

For completeness, in Sec. II additional studies of coherence threshold calculation are discussed. Then the experiment and core noise related coherence definitions are discussed. Next the statistical estimate of the coherence threshold is discussed. Then the aligned and un-aligned coherence method and results are presented. In presenting the results, first some simulation results are presented. Then, some experimental Pratt \& Whitney PW4098 turbofan engine results are analyzed. Next, the results are discussed and conclusions are presented.

\section{OTHER THRESHOLD COHERENCE STUDIES}

A slightly different aligned and un-aligned coherence procedure was used by Gross et al. ${ }^{19}$ in a study of corticomuscular synchronization during isometric muscle contraction in humans as revealed by magnetoencephalography. After calculating the aligned and un-aligned coherence, the confidence limit $\gamma_{n n}^{2}(1-\alpha)$ is estimated as a value under which lies $(1-\alpha) 100 \%$ of the values of the estimated MSC function in the frequency range used. Gross used a 50\% overlap.

The procedure used by Gross et al. ${ }^{19}$ was examined by Bortel and Sovka ${ }^{33}$ in a study of an approximation of the statistical distribution of the MSC function estimated with segment overlapping. They are unhappy with this estimation procedure. First, since it is based on the estimation of a $1-\alpha$ quantile, which is an imprecise operation for which there are many procedures, it can lead to different confidence limit estimates. Second, since various time delays $D$ produce different MSC functions this can also lead to different confidence limit estimates. Bortel and Sovka ${ }^{33}$ go on to develop an approximation to the statistical distribution of the MSC function with segment overlapping and to develop a procedure for confidence interval estimation. Bortel and Sovka ${ }^{33}$ suggest that the value $n_{d}$ used in calculating the statistical coherence threshold corresponds to the number of degrees of freedom, $L$. They then speculate that the statistical distribution of his time series is similar to that used in developing the statistical threshold and that details like the amount of overlap and the window can be accounted for by the proper choice of the number of degrees of freedom, $L$. Next, estimation equations are derived for $L$.

A surrogate data analysis procedure studied by Faes et $a l .{ }^{12}$ has been used in biomedical engineering studies to develop a threshold for the coherence of two uncorrelated time histories. The paper by Faes et al. examines three procedures for generating surrogate series. The method of surrogate data generates a set of surrogate series mimicking some properties of the original series but being otherwise uncoupled by for example randomly shuffling sample blocks. Using a coherence of the surrogate data, the confidence limit $\gamma_{n n}^{2}(1-\alpha)$ is estimated as a value under which lies $(1-\alpha) 100 \%$ of the values of the estimated MSC function in the frequency range used. Consequently, the surrogate data procedure also depends on the method used for

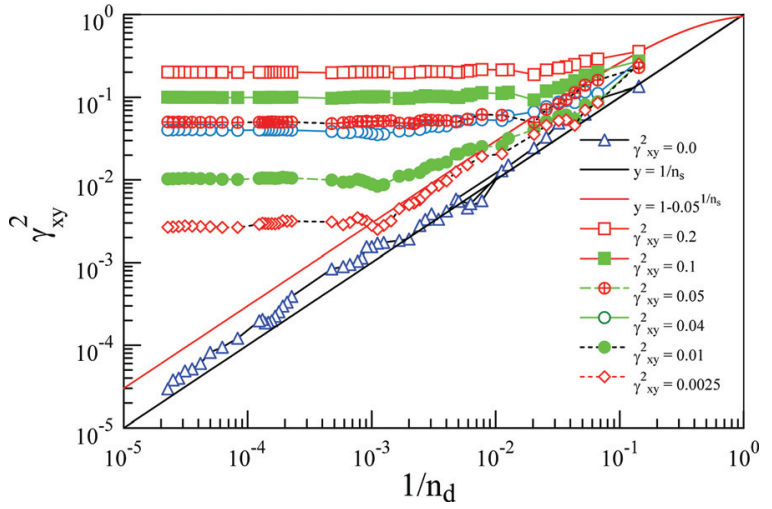

FIG. 1. (Color online) Simulation result summary.

calculating the coherence such as the amount of sample overlap. However, it cannot identify tones since the random shuffling of data sample blocks should remove periodic information. In addition, the method has a similar lack of precision as aligned and un-aligned coherence method, since different procedures for generating the surrogate series might give different results and many procedures are available for calculating the confidence limit.

In this paper, the un-aligned and un-aligned coherence procedure and the threshold statistical procedure using $L=n_{d}$ and $L=2 n_{d}$ are examined and compared. The surrogate data analysis procedure studied by Faes et al. ${ }^{12}$ and the procedure suggested by Bortel and Sovka ${ }^{33}$ were not examined.

\section{EXPERIMENT}

To demonstrate the usefulness of the proposed aligned and un-aligned coherence method, pressure measurements made in a Pratt \& Whitney PW4098 combustor will be used. The measurements were made in a study of aircraft turbofan engine core noise conducted as part of the NASA EVNRC Program at test stand C-11 in West Palm Beach, Florida. The engine had an attached acoustic inflow control device. A photograph of the test site appears in Fig. 1 of Ref. 11. The combustor was instrumented with two pressure sensors. One water cooled combustor pressure sensor was at $127^{\circ}$ (sensor 1) and the other was at $337^{\circ}$ (sensor 2). A photograph of the mounted hardware is shown in Fig. 2 of Ref. 11. Combustor pressure sensor angles are measured clockwise from top dead center viewed from the rear or exhaust section of the engine. The spectral estimate parameters are shown in Table I. The signal processing procedure used is discussed in Sec. IV. The far-field microphones used herein are on a 150 foot radius at angles of $100^{\circ}$ (sensor 3), $110^{\circ}$ (sensor 4), $120^{\circ}$ (sensor 5), and $130^{\circ}$ (sensor 6 ) measured from the inlet. The acoustic arena and microphone array are shown in Fig. 3 of Ref. 11.

\section{CORE NOISE STUDY SYSTEM CONCEPTS}

The procedure discussed herein involves the following items:

(1) The estimated MSC function, $\hat{\gamma}_{x y}^{2}(f)$ :

First, the MSC function will be discussed. Then, the estimation procedure will be discussed. 

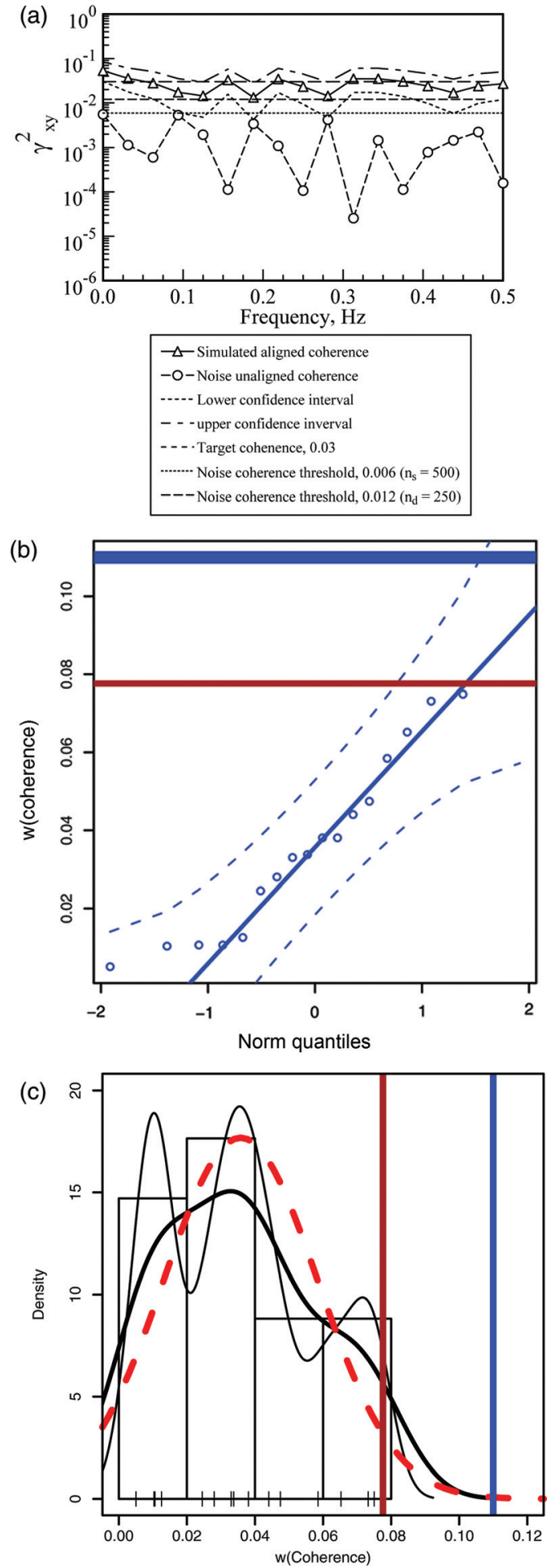

FIG. 2. (Color online) Plots show target coherence, measured aligned coherence, the confidence interval, the un-aligned noise coherence, the estimated upper $95 \%$ confidence limit threshold for a $50 \%$ overlap $\hat{\gamma}_{x_{n} y_{n}}^{2}\left(f, P_{I}=0.95\right.$, $\left.n_{s}=500\right) \approx 0.006$, and based on the assumption of independent records the estimated upper $95 \%$ confidence limit threshold $\hat{\gamma}_{x_{n} y_{n}}^{2}\left(f, P_{I}=0.95\right.$, $\left.n_{d}=250\right) \approx 0.012$. (a) Simulation using target coherence $\left\{\hat{\gamma}_{x_{n} y_{n}}^{2}\right\}^{T}=0.03$. (b) Norm quantile comparison of un-aligned noise coherence. (c) Histogram of unaligned noise coherence.

The MSC function, $\hat{\gamma}_{x y}^{2}(f)$, calculated using the complex cross-spectral density, $\hat{G}_{x y}(f)$, and two auto-spectral densities at frequency $f, \hat{G}_{x x}(f)$ and $\hat{G}_{y y}(f)$, is defined by
TABLE I. Spectral estimate parameters.

\begin{tabular}{lc}
\hline \hline Parameter & Value \\
\hline Segment/block length, i.e., Data points & 4096 \\
per segment/block, $N P$ & \\
Sample rate, $r$, samples/s & 48000 \\
Segment length, $T_{d}=N P / r, \mathrm{~s}$ & 0.08533 \\
Sampling interval, $\Delta t=1 / r, \mathrm{~s}$ & $2.0833 \times 10^{-5}$ \\
Frequency step, $\Delta f=B_{e}=1 / T_{\mathrm{d}}, \mathrm{Hz}$ & 11.718 \\
Upper frequency limit, $f_{c}=1 / 2 \Delta \mathrm{t}=r / 2, \mathrm{~Hz}$ & 24000 \\
number of frequencies, $L y=f_{c} / \Delta f=N P / 2$ & 2048 \\
Time delay to far field, $\tau=6323 / 48000, \mathrm{~s}$ & 0.1317 \\
number of independent segments/blocks & 234 \\
Overlap & 0.50 \\
Sample length, s & 20 \\
Spectral window & Rectangular \\
\hline \hline
\end{tabular}

$$
\hat{\gamma}_{x y}^{2}(f)=\frac{\left|\hat{G}_{x y}(f)\right|^{2}}{\hat{G}_{x x}(f) \hat{G}_{y y}(f)} .
$$

The MSC function is discussed by Bendat and Piersol, ${ }^{15}$ Carter ${ }^{7}$ Halliday et al. ${ }^{8}$ and Jenkins and Watts ${ }^{16}$ where it is called the squared coherency spectrum.

The MSC function estimation procedure used herein are based on signal processing algorithms developed by Stearns and David. ${ }^{3}$ They refer to their spectralestimation code as using a periodogram averaging method. The algorithm is also referred to a being based on Welch's method $\left(\right.$ Welch $\left.^{1}\right)$ and as a weighted overlapped segment averaging (WOSA) method (Carter et $_{\text {al. }}{ }^{18}$ ).

Two additional characteristics of the MSC estimation process is its sensitivity to the alignment or translation of the time series and the inability to observe tones mixed with broadband noise in the spectrum. These points will be discussed later. One of the objectives of this report is to point out how deliberate misalignment of the time series can enable one to observe tones in the spectrum.

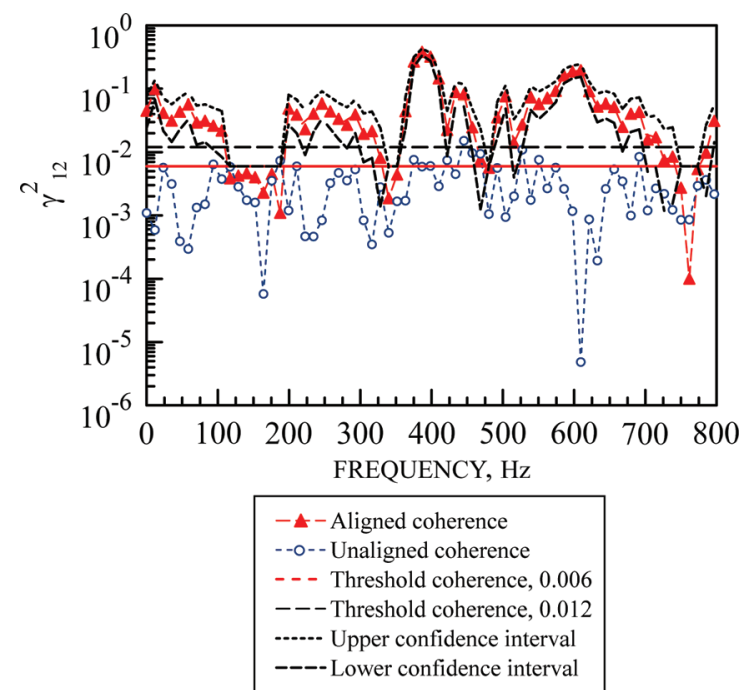

FIG. 3. (Color online) Aligned and un-aligned coherence between combustor pressure sensors 1 and 2 . The broken horizontal line is $\hat{\gamma}_{x_{n} y_{n}}^{2}(f$, $\left.P_{I}=0.95, n_{d}=250\right) \approx 0.012$. The unbroken horizontal line is $\hat{\gamma}_{x_{n} y_{n}}^{2}(f$, $\left.P_{I}=0.95, n_{s}=500\right) \approx 0.012$. N1 Corr $=1622$.

Jeffrey Hilton Miles: Detection of turbofan engine combustion noise 
(2) The spectrum based on the three signal magnitude coherence function developed by Chung et al. ${ }^{21}$ and Chung ${ }^{22}$ for flow noise rejection is (also see Appendix B of Ref. 11)

$\hat{G}_{u_{1} u_{1}}(f)=\hat{G}_{y_{1} y_{1}}(f) \frac{\left|\hat{\gamma}_{y_{1} y_{2}}(f)\right|\left|\hat{\gamma}_{y_{1} y_{3}}(f)\right|}{\left|\hat{\gamma}_{y_{2} y_{3}}(f)\right|}$.

(3) The coherent output power spectrum function discussed by Bendat and Piersol ${ }^{15}$ (also see Appendix C of Ref. 11)

$\hat{W}_{s_{F} s_{F}}(f)=\hat{\gamma}_{x y}^{2}(f) \hat{G}_{y y}(f)$.

\section{TRADITIONAL VIEWPOINT}

First, the traditional view on measuring coherence will be presented to pinpoint the "blind spots." A good place to start is a statement about the proper analysis procedures to obtain a coherence measurement as stated by Piersol: ${ }^{34}$

(1) The instrumentation is calibrated to eliminate relative phase errors in the measured signals.

(2) The received signals are aligned as required to eliminate time delay bias errors in the coherence calculation.

(3) The number of averages $n_{d}$ is sufficiently large to suppress small sample bias errors in the coherence calculations.

\section{STATISTICAL ESTIMATES OF COHERENCE}

\section{A. Confidence interval}

A significant limitation to the application of the MSC estimate is that for coherence values smaller than some fairly large value (like 0.1) and for large sample sizes, the confidence interval is not available. For values greater than some small value (like 0.1), the confidence interval is only given approximately (Bendat and Piersol, ${ }^{35}$ Brillinger, ${ }^{9}$ and Carter $^{7,18}$ ). A procedure calculating the exact confidence interval is discussed by Wang et al. ${ }^{36,37}$ and by Miles. ${ }^{11}$ Consequently, if one calculates the upper and lower confidence interval one can determine the significance of the estimation and the significance of the differences of MSC values at different frequencies. Confidence limits are shown in Fig. 4 of Ref. 11 for $n_{d}=600$. The confidence limits are shown on linear plot as they are usually shown in Fig. 4(a) of Ref. 11. The confidence limits are plotted in a more realistic manner in Fig. 4(b) of Ref. 11 on a logarithmic plot, which demonstrates clearly that for a given value of $n_{d}$, the confidence interval becomes larger as the value of $\hat{\gamma}_{x y}^{2}$ is reduced.

\section{B. Statistical estimate of coherence threshold of independent processes}

In considering the MSC of two stationary Gaussian random processes, it is often stated that the MSC will be zero when the processes are independent and do not have a linear time-invariant relationship. However, the estimated MSC of two independent processes is never zero but has some small numerical value at each frequency. The only statement that can be made is that if the true MSC value is $0,|\gamma|=0$, the expected value is in a certain confidence interval with a

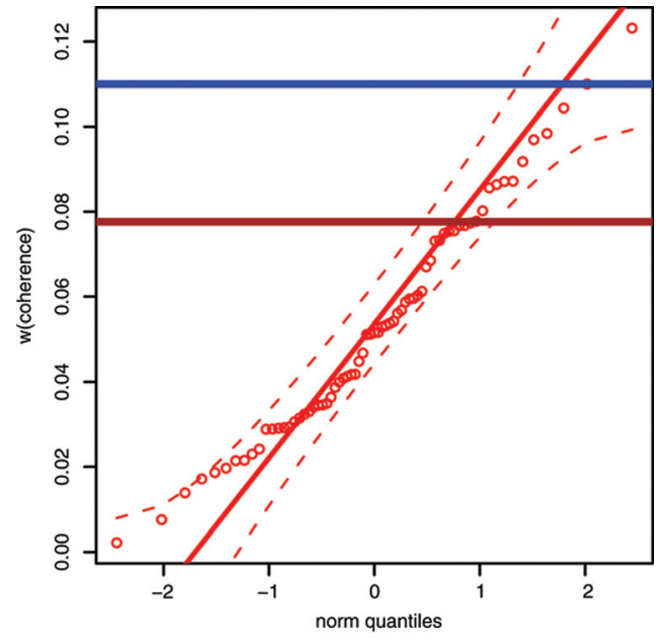

FIG. 4. (Color online) Quantile comparison plot of unaligned coherence between combustor pressure sensor (1) and (2) with normal distribution. The broken lines give a pointwise $95 \%$ confidence envelope around the fitted solid line. The upper horizontal line at $w(\sqrt{0.012})=\tanh ^{-1}(0.10954)$ $\approx 0.109$ represents a statistical coherence threshold of $\hat{\gamma}_{x_{n} y_{n}}^{2}\left(f, P_{I}\right.$ $\left.=0.95, n_{d}=250\right) \approx 0.012$. The lower horizontal line at $w(\sqrt{0.006})$ $=\tanh ^{-1}(0.07746) \approx 0.0776$ represents statistical coherence threshold of $\hat{\gamma}_{x_{n} y_{n}}^{2}\left(f, P_{I}=0.95, n_{s}=500\right) \approx 0.006 . \mathrm{N} 1$ Corr $=1622$.

certain probability. The threshold value of this confidence interval will be called $E_{\mathrm{I}}$.

The conditional probability of the estimated value of the MSC, $|\hat{\gamma}|^{2}$, given the true MSC is zero, $|\gamma|^{2}=0$, and the number of independent segments/blocks, $n_{d}$, can be calculated from as

$$
p\left(\left.|\hat{\gamma}|^{2}\left|n_{d},\right| \gamma\right|^{2}=0\right)=\left(n_{d}-1\right)\left(1-|\hat{\gamma}|^{2}\right)^{n_{d}-2} .
$$

This equation is given by Brillinger ${ }^{9}$ and is discussed by Miles. ${ }^{11}$

Then the probability, $P_{I}$, that $|\hat{\gamma}|^{2}$ is in the interval $0<|\hat{\gamma}|^{2}<E_{I}$ can be calculated as

$$
\begin{aligned}
\hat{C} & =\mid \hat{\gamma}^{2} \\
P_{I} & =\int_{0}^{E_{I}} p\left(\left|\hat{\gamma}^{2}\right| n_{d},|\gamma|^{2}=0\right) d|\hat{\gamma}|^{2} \\
& =\int_{0}^{E_{I}}\left(n_{d}-1\right)(1-\hat{C})^{n_{d}-2} d \hat{C} \\
& =-\left.(1-\hat{C})^{n_{d}-1}\right|_{0} ^{E_{I}} \\
& =-\left(1-E_{I}\right)^{n_{d}-1}+1 .
\end{aligned}
$$

Thus, the cumulative distribution derived using the probability density function of the coherence derived by Goodman ${ }^{38}$ has been used to determine a $P_{I}$ percent confidence interval, if the true MSC is zero. This is given by

$$
\hat{\gamma}_{x_{n} y_{n}}^{2}\left(f, P_{I}, n_{d}\right)=E_{I}=1-\left(1-P_{I}\right)^{1 /\left(n_{d}-1\right)},
$$

where $n_{d}$ is the number of independent segments used in the spectrum calculations and the interval is between 0 and $\hat{\gamma}_{x_{n} y_{n}}^{2}\left(f, P_{I}, n_{d}\right)$. This relationship is tabulated in Table III by Miles ${ }^{11}$ for probability values, $P_{I}$, of $80 \%, 90 \%, 95 \%$, and 
99\% and is shown in Fig. 1. This statistical test for independence using Eq. 7 is discussed by Carter, ${ }^{6,7}$ who proposes using $\hat{\gamma}_{x_{n} y_{n}}^{2}\left(f, P_{I}, n_{d}\right)$ as a threshold value below which coherence estimates are not presented to a human decision maker looking at an extensive volume of data collected for brain wave analysis or sonar or radar signal analysis. This equation is also given by Gosselin, ${ }^{39}$ Halliday et al., ${ }^{8}$ and Brillinger. ${ }^{9}$ Halliday suggests one should plot the value of $\hat{\gamma}_{x_{n} y_{n}}^{2}\left(f, P_{I}, n_{d}\right)$ calculated from Eq. (7), i.e., under the hypothesis of independence on each coherence plot. He states that estimated values of coherence lying below this line can be taken as evidence to indicate that the coherence is zero at that frequency. This opinion is shared by Nuttall ${ }^{40}$ who thinks this is the standard result for a true coherence of zero. However, the author thinks it may indicate that not enough segments/blocks have been used to determine the coherence. One can state with more confidence that values above the $\hat{\gamma}_{x_{n} y_{n}}^{2}\left(f, P_{I}, n_{d}\right)$ line are not from two independent stationary random Gaussian series. Note that this statement is valid independent of the magnitude of $\hat{\gamma}_{x_{n} y_{n}}^{2}\left(f, P_{I}, n_{d}\right)$. In this paper, we examine whether one should calculate the coherence threshold using

$$
\hat{\gamma}_{x_{n} y_{n}}^{2}\left(f, P_{I}, n_{s}\right)=1-\left(1-P_{I}\right)^{1 /\left(n_{s}-1\right)},
$$

where with a sample overlap of $50 \% n_{s}=2 n_{\mathrm{d}}$. In the figure legends, the notation for the threshold coherence drops the accents and becomes simply $\gamma_{n n}^{2}(n)$ where the $n$ value in parentheses indicates one is using the number of independent samples $n_{d}$ or the number of overlapped samples $2 n_{d}$ and the confidence interval probability, $P_{I}$, is $95 \%$.

\section{COHERENCE TRANSFORM}

When the transformation

$$
\begin{aligned}
z & =\frac{1}{2} \ln \left(\frac{(1+\sqrt{(x)})}{(1-\sqrt{(x)})}\right) \\
& =\tanh ^{-1}(\sqrt{x})
\end{aligned}
$$

(termed the Fisher $\mathrm{z}$-transformation ${ }^{20}$ ) is applied to the ordinary correlation coefficient, one obtains a variable which is normally distributed to a very high degree of accuracy. Consequently, the random variable $w$

$$
\begin{aligned}
w\left(\hat{\gamma}_{x y}\right) & =\frac{1}{2} \ln \left(\frac{\left(1+\hat{\gamma}_{x y}\right)}{\left(1-\hat{\gamma}_{x y}\right)}\right) \\
& =\tanh ^{-1}\left(\hat{\gamma}_{x y}\right)
\end{aligned}
$$

is to a close approximation, Gaussian with a distribution $N\left(\mu, \sigma^{2}\right) .{ }^{41}$ The $w\left(\hat{\gamma}_{x y}\right)$ transforms of the estimated nonaligned coherence functions calculated herein will be compared to normal distributions using histograms and quantile-quantile (Q-Q) plots, which are the standard statistical analysis tools. ${ }^{42}$

\section{COHERENCE AND SIMULATION METHODS}

\section{A. Simulation procedure}

Many assessments of the validity of the statistical estimates of coherence have been made using simulations. The probability density function of the coherence derived by Goodman $^{38}$ was derived for Gaussian data. Foster and Guinzy $^{43}$ tested this distribution by means of Monte Carlo experiments for validity and robustness (insensitivity to the Gaussian assumption) and it passed the tests. Coherence function bias and confidence intervals were studied using Monte Carlo methods by Benignus. ${ }^{44}$

The probability density function of the coherence derived by Goodman ${ }^{38}$ does not take into account the use of overlapping data. Empirical simulation results by Carter $^{7}$ and Carter et al. ${ }^{18}$ show a decrease in bias and variance of the estimator with increasing overlap and suggest a $50 \%$ overlap as being highly desirable.

Furthermore, since the probability density function of the coherence derived by Goodman ${ }^{38}$ is independent of the computer code time series analysis implementation comparing bias and variance simulation results can provide some confidence in the analysis procedure. Simulations were done for a range of target coherence values and a range of independent segments/blocks using

$$
\begin{aligned}
& x_{n}(t)=n_{3}+\alpha n_{1}, \\
& y_{n}(t)=n_{3}+\alpha n_{2}, \\
& z_{n}(t)=n_{3},
\end{aligned}
$$

where $n_{1}, n_{2}$, and $n_{3}$ are independent Gaussian random time series. Results will be presented in Figs. 1 and 2 .

The time series are selected so that

$$
\begin{aligned}
& G_{n_{3} n_{3}}=G_{n_{1} n_{1}}=G_{n_{2} n_{2}}=G_{s s}, \\
& G_{n_{1} n_{2}}=G_{n_{3} n_{1}}=G_{n_{3} n_{2}}=0 .
\end{aligned}
$$

Taking the Fourier transform of $x_{n}$ yields

$$
X_{x_{n}}(f)=X_{n_{3}}(f)+\alpha X_{n_{1}}(f) .
$$

The spectrum $G_{x_{n} x_{n}}$ is then

$$
\begin{aligned}
G_{x_{n} x_{n}} & =X_{x_{n}}(f) X_{x_{n}}^{*}(f) \\
& =\left(X_{n_{3}}(f)+\alpha X_{n_{1}}(f)\right)\left(X_{n_{3}}(f)+\alpha X\left(n_{1}\right)(f)\right)^{*} \\
& =G_{n_{3} n_{3}}+\alpha^{2} G_{n_{1} n_{1}} .
\end{aligned}
$$

We then also have $G_{y_{n} y_{n}}=G_{n_{3} n_{3}}+\alpha^{2} G_{n_{2} n_{2}}$ and

$$
\begin{aligned}
G_{x_{n} x_{n}} & =G_{n_{3} n_{3}}, \\
G_{x_{n} z_{n}} & =G_{n_{3} n_{3}}, \\
G_{y_{n} z_{n}} & =G_{n_{3} n_{3}}, \\
G_{z_{n} z_{n}} & =G_{n_{3} n_{3}} .
\end{aligned}
$$

Then the target MSC is a function of $\alpha$ given by

$$
\left\{\hat{\gamma}_{x_{n} y_{n}}^{2}(f)\right\}^{T}=\frac{\left|\hat{G}_{x_{n} y_{n}}\right|^{2}}{\hat{G}_{x_{n} x_{n}} \hat{G}_{y_{n} y_{n}}}=\frac{1}{\left(1+\alpha^{2}\right)^{2}}
$$

and

$$
\left\{\hat{\gamma}_{x_{n} z_{n}}^{2}(f)\right\}^{T}=\left\{\hat{\gamma}_{y_{n} z_{n}}^{2}(f)\right\}^{T}=\frac{1}{\left(1+\alpha^{2}\right)} .
$$


These expressions are independent of frequency. A similar approach is used to create target simulations by Carter et al. ${ }^{18}$ and Guinzy. ${ }^{43}$

\section{B. Simulation results}

Results of a typical simulation using a target coherence of $\left\{\hat{\gamma}_{x_{n} y_{n}}^{2}(f)\right\}^{T}=0.03$ are shown in Fig. 2(a). The simulations are done with $n_{d}=250$ with a $50 \%$ overlap, so $n_{\mathrm{s}}=500$. The coherence is low, so a logarithmic coherence scale is used in Fig. 2(a). The target coherence is shown by the heavy dashed line. The plot shows the estimated or measured coherence, $\hat{\gamma}_{x y}^{2}(f)$, as triangles and an estimated un-aligned signal coherence, $\left|\hat{\gamma}_{x_{n} y_{n}}^{2}(f)\right|$ as circles for $n_{s}=500$. The upper and lower confidence interval is shown as dashed lines. In addition, the lower horizontal statistical coherence threshold line $\hat{\gamma}_{x_{n} y_{n}}^{2}\left(f, P_{I}=0.95, n_{s}=500\right) \approx 0.006$ and the higher horizontal statistical coherence threshold line $\hat{\gamma}_{x_{n} y_{n}}^{2}\left(f, P_{I}=0.95\right.$, $\left.n_{s}=250\right) \approx 0.012$ are shown. For this case, the estimated un-aligned signal coherence, $\left|\hat{\gamma}_{x_{n} y_{n}}^{2}(f)\right|$, is less than the lower statistical coherence threshold $\left(\hat{\gamma}_{x_{n} y_{n}}^{2}\left(f, P=0.95, n_{s}=500\right)\right.$ $\approx 0.006$ ) and the measured coherence is well above both statistical coherence thresholds. Consequently, the coherence while low is significant. The lower statistical coherence threshold line traces the tops of the estimated un-aligned coherence curve. The upper statistical coherence threshold line at $\hat{\gamma}_{x_{n} y_{n}}^{2}\left(f, P_{I}=0.95, n_{s}=250\right) \approx 0.012$ is at a value greater than that of the estimated un-aligned signal coherence, $\left|\hat{\gamma}_{x_{n} y_{n}}^{2}(f)\right|$, which has values less than 0.006 .

The distribution of the un-aligned noise coherence plotted in Fig. 2(a) was examined to determine if it had a normal distribution using two procedures. A norm quantile comparison plot of the Fisher z-transformed un-aligned noise coherence using Eq. (9) is shown in Fig. 2(b). The broken lines give a pointwise $95 \%$ confidence envelope around the fitted solid line. Horizontal lines corresponding to Fisher z-transformed un-aligned threshold coherence values of 0.006 and 0.012 are also shown. The upper horizontal line in Fig. 2(b) at $w(\sqrt{0.012})=\tanh ^{-1}(0.1095) \approx 0.109$ represents an estimate of the upper $95 \%$ threshold coherence confidence limit of the un-aligned coherence based on 250 independent samples. The lower horizontal line at $w(\sqrt{0.006})$ $=\tanh ^{-1}(0.07746) \approx 0.077$ represents an estimate of the upper $95 \%$ threshold coherence confidence limit of the unaligned coherence based on 500 samples. This line is seen to provide a better coherence threshold for the $95 \%$ confidence interval and to be in good agreement with the estimated unaligned signal coherence values, $\left|\hat{\gamma}_{x_{n} y_{n}}^{2}(f)\right|$, shown in Fig. 2(b). The coherence threshold calculated with $n_{d}=250$ is larger than necessary.

A corresponding histogram plot of the Fisher z-transformed estimated un-aligned signal coherence values, $\left|\hat{\gamma}_{x_{n} y_{n}}^{2}(f)\right|$ is shown in Fig. 2(c). Due to a lack of observations only four bins are used. Also shown are non-parametric kernel-density estimates for the distribution of coherence, using the default bandwidth (heavier line), which produces a smoother density estimate, and half the default bandwidth (lighter line), which produces a rougher density estimate. A normal distribution is shown as a dashed curve. A one- dimensional scatter plot is shown at the bottom of the graph, using a vertical bar as the plotting symbol, with the horizontal location given by coherence. On this plot vertical lines indicate estimates of the upper $95 \%$ statistical threshold coherence confidence limit calculated with $n_{d}=250$ which produces a line at $w(0.012) \approx 0.109$ and $n_{s}=500$ which produces a line at $w(0.006) \approx 0.077$. The line produced with $n_{s}=500$ is again seen to produce a better statistical coherence threshold value.

The results of a great many simulations are shown in Fig. 1 where average MSC values, $\bar{\gamma}_{x y}^{2}$, are presented as a function of the number of independent segments/blocks, $n_{d}$.

Each simulation resembles the one shown in Fig. 2(a) and is designed for a particular target value. We use as a measure of convergence to a particular target value

$$
\bar{\gamma}_{x y}^{2}=\frac{1}{n} \sum_{i=0}^{n-1} \hat{\gamma}_{x y}^{2}\left(f_{i}\right)
$$

Simulations with a range of target MSC values calculated using Eqs. (9) and (10) are shown. The symbols trace each target value. The target values are given in the legend. In addition, simulation results for the mean value when two independent simulated random Gaussian time histories are used to calculate $\hat{\gamma}_{x_{n_{1}} y_{n_{2}}}^{2}(f)$ are shown as black triangles and identified as $\gamma_{x y}^{2}=0$ in the legend.

The results show that a coherent pair of signals will have a coherence that resembles the coherence of random noise if the number of segments/blocks, $n_{d}$, is too small. For example, for $n_{d}=100$, one would see an indication of MSC $=0.01$ but not of MSC $=0.0025$. Consequently, the value $n_{d}$ controls not only the bias and variance of the coherence but also its existence. Consequently, for a given measurement of coherence using a given value of $n_{d}$ one can say that a coherence may not be observed if there is a $P$ percent probability that it is less than $\hat{\gamma}_{x_{n} y_{n}}^{2}\left(f, P_{I}, n_{d}\right)=E_{I}=1-\left(1-P_{I}\right)^{1 /\left(n_{d}-1\right)}$ Eq. (7). This curve is identified as $y=1-0.05^{x}$ in the legend box. This curve is shown in the figure as a thin line for a $95 \%$ confidence interval. In addition, the $\gamma_{x y}^{2}=1 / n_{d}$ line is shown as a diagonal black line identified as $y=x$ in the legend. This line corresponds to the expected or mean value of $|\bar{\gamma}|_{x y}^{2}$ for $|\bar{\gamma}|_{x y}^{2}=0$ as discussed in Ref. 10 [see Eq. (12)]. This curve also corresponds to the one Tukey ${ }^{45}$ suggested as discussed in Ref. 11. The estimated mean value of $|\bar{\gamma}|_{n_{1} n_{2}}^{2}(f)$ shown by the black triangles is close to this line. However, the $95 \%$ confidence interval provides a better number to be larger than, when one wishes to identify a small coherence value as being significant.

The simulations showed that with a Gaussian distribution, the coherence threshold calculated using the un-aligned coherence with a $50 \%$ overlap is similar to the statistical coherence threshold calculated with $n_{s}=2 n_{d}$.

\section{ALIGNED AND UN-ALIGNED COHERENCE}

The method of aligned and un-aligned coherence is based on the following two characteristics of the average periodogram process when it is used to calculate the MSC:

(1) When the deliberate time delay shift interval, $\tau_{1}$, exceeds the sample record length, $T_{d}$, the coherence of the 
random process, $\hat{\gamma}_{x y}^{2}(f)$, is the coherence of two independent random signals $\left|\hat{\gamma}_{x_{n} y_{n}}^{2}(f)\right|$.

(2) If the signals contain tones using a deliberate time delay shift, $\tau_{1}$, greater than the sample record length, $T_{d}$, does not change the coherence of the tones and permit the estimation of hidden periodicities, concealed spectral lines, and undamped sinusoids. These items are generally referred to as tones herein.

The deliberate time delay shift interval is sometimes expressed herein in terms of the number of data points contained in the time delay shift interval, $D$, which is the product of the sample rate, $r$, and the time delay shift, $\tau_{1},\left(D=r \tau_{1}\right)$. Spectral estimate parameters used herein are shown in Table I. For the data processed herein, the length of the sample record or segment interval, $T_{s}$, is $T_{d}=N P / r=0.08533 \mathrm{~s}$ since the number of data points contained in a segment/block is $N P=4096$ and the sampling rate, $r$, is $r=48000$ samples/s. The necessary condition to get nonalignment can be expressed as deliberately displacing one time series with respect to another by shifting it an amount $D$ such that $D>N P$ or by displacing it in time by an amount $\tau_{1}$ where $\tau_{1}>T_{d}$.

\section{EXPERIMENTAL RESULTS}

\section{A. Combustor pressure sensor coherence}

Logarithmic plots of aligned coherence, $\hat{\gamma}_{x y}^{2}(f)$ (shown by filled triangles), and un-aligned coherence, $\left|\hat{\gamma}_{x_{n} y_{n}}^{2}(f)\right|$ (shown by circles), between the two combustor pressure sensors are shown in Fig. 3 for a typical test conditions (N1 Corr $=1622$ ). Also shown are the confidence intervals for the aligned coherence. Note that as indicated in Fig. 4(b) of Ref. 11 , the confidence interval is narrow for coherence values near 1 and becomes much larger for lower coherence values. In addition, the lower horizontal statistical coherence threshold line $\hat{\gamma}_{x_{n} y_{n}}^{2}\left(n_{s}\right)=\hat{\gamma}_{x_{n} y_{n}}^{2}\left(f, P_{I}=0.95, n_{s}=500\right) \approx 0.006$ and the higher horizontal statistical coherence threshold line $\hat{\gamma}_{x_{n} y_{n}}^{2}\left(n_{d}\right)=\hat{\gamma}_{x_{n} y_{n}}^{2}\left(f, P_{I}=0.95, n_{s}=250\right) \approx 0.012$ calculated from Eq. (7) are shown.

As previously mentioned, the PW4098 combustor pressure sensor cross-spectra amplitude and phase plots resembled the cross-spectra used in the acoustic modal analysis of an YF102 combustor installed in a ducted test rig, which was conducted by Karchmer. ${ }^{24}$ This suggested that the observed structure in the coherence was due to a modal pattern. Consequently, using a model of the physics of pressure waves propagating in annular ducts as discussed by Tyler and Sofrin ${ }^{25}$ in a treatment of axial flow compressor noise, a procedure was developed that uses the available combustor pressure sensor data to obtain a descriptions of the combustion modes in the annular combustor. The model is discussed by Miles. ${ }^{26}$ Results from this work were used to select the signals to be analyzed and to interpret the results in an attempt to identify combustion noise in the farfield data. These broad peaks and dips in the coherence due to combustion duct modes are seen in Fig. 3. The coherent plane wave mode as determined by a model analysis is apparent in Fig. 3 between 0 and $200 \mathrm{~Hz}$ at 1622 rpm (N1 Corr.).

Note in Fig. 3, the aligned coherence, $\hat{\gamma}_{x y}^{2}(f)$ (shown by diamonds) and the un-aligned coherence values $\left|\hat{\gamma}_{x_{n} y_{n}}^{2}(f)\right|$ (shown by circles) are well separated. The aligned coherence, $\hat{\gamma}_{x y}^{2}(f)$, is well above both of the statistical threshold coherence horizontal lines $\left[\hat{\gamma}_{x_{n} y_{n}}^{2}(f)\right.$ and $\left.\hat{\gamma}_{x_{n} y_{n}}^{2}\left(n_{d}\right)\right]$ and above the estimated un-aligned coherence, $\left|\hat{\gamma}_{x_{n} y_{n}}^{2}(f)\right|$, except in a region between 400 and $500 \mathrm{~Hz}$. This bump appears to be a tone since it appears in both the aligned and un-aligned coherence.

The same two procedures that were applied to the simulated time series are applied to the engine noise time series analysis to determine if the un-aligned noise coherence shown in Fig. 3 has a normal distribution. A norm quantile comparison plot of the Fisher z-transformed un-aligned noise coherence using Eq. (9) is shown in Fig. 4 for a typical engine operating condition $(\mathrm{N} 1 \mathrm{Corr}=1622 \mathrm{rpm})$. The broken lines give a pointwise $95 \%$ confidence envelope around the fitted solid line. Horizontal lines corresponding to Fisher z-transformed un-aligned noise coherence threshold values of 0.006 and 0.012 are also shown. The upper horizontal line in Fig. 4 at $w \sqrt{0.012}$ $=\tanh ^{-1}(0.1095) \approx 0.109$ represents an estimate of the upper $95 \%$ confidence limit of the un-aligned coherence threshold based on 250 independent samples. The lower horizontal line at $w \sqrt{0.006}=\tanh ^{-1}(0.07746) \approx 0.077$ in these figures represents an estimate of the upper $95 \%$ confidence limit of the unaligned coherence threshold based on 500 samples. However, for this turbofan engine data case the upper horizontal line based on $n_{d}=250$ provides a better coherence threshold.

The corresponding histogram plot of the Fisher z-transformed un-aligned noise coherence is shown in Fig. 5 for the N1 Corr $=1622 \mathrm{rpm}$ case. Also shown are non-parametric kernel-density estimates for the distribution of coherence, using the default bandwidth (heavier line), which produces a smoother density estimate, and half the default bandwidth

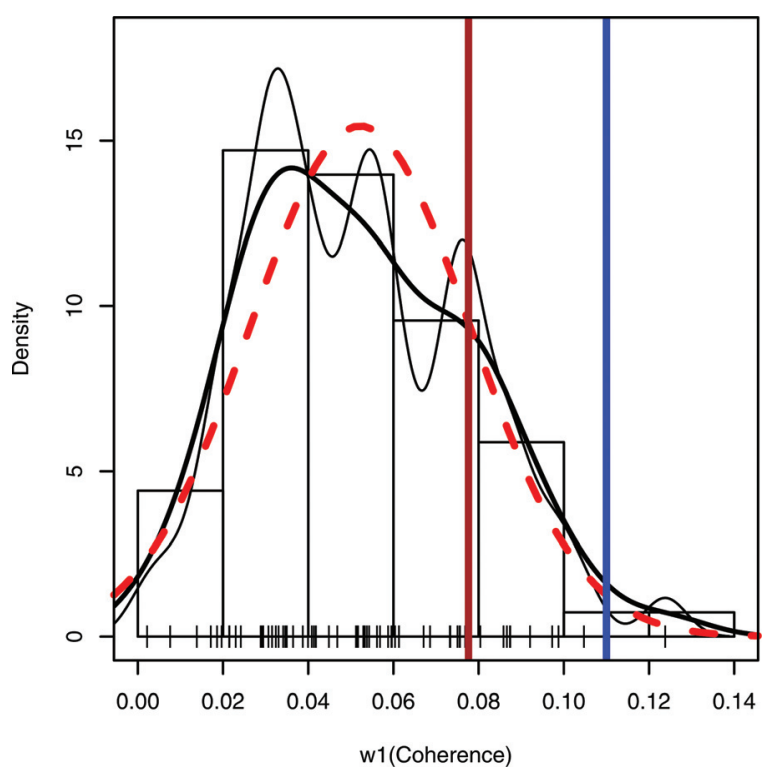

FIG. 5. (Color online) Histogram of un-aligned coherence between combustor pressure sensors 1 and 2 . Vertical lines $w(\sqrt{0.006})=\tanh ^{-1}(0.07746)$ $=0.0776$ and $w(\sqrt{0.012})=\tanh ^{-1}(0.10954)=0.109$ represent statistical coherence thresholds of $\hat{\gamma}_{x_{n} y_{n}}^{2}\left(f, P_{I}=0.95, n_{s}=500\right) \approx 0.006$ and $\hat{\gamma}_{x_{n} y_{n}}^{2}$ $\left(f, P_{I}=0.95, n_{d}=250\right) \approx 0.012$ Non-parametric kernel-density estimates for the distribution of coherence, using the default bandwidth (heavier line) and half the default bandwidth (lighter line). A one-dimensional scatter plot at the bottom of the graph uses a vertical bar to indicate the horizontal position of each coherence magnitude value. N1 Corr $=1622$. 


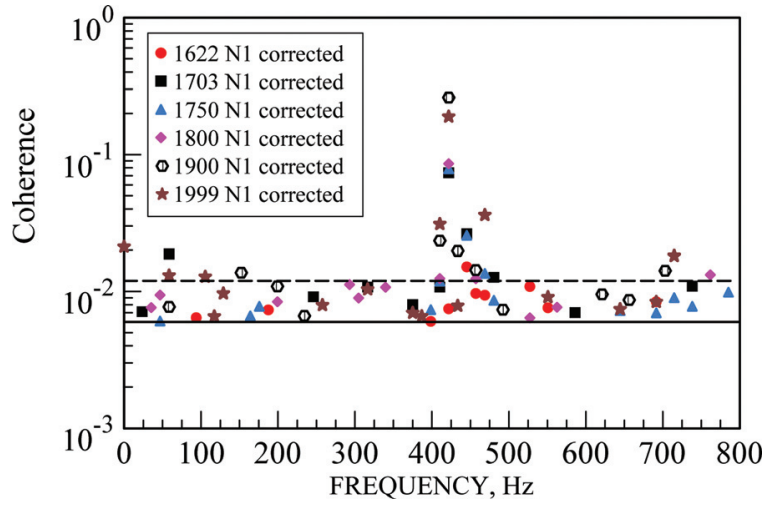

FIG. 6. (Color online) Scatter plot of culled un-aligned coherence points from coherence between combustor pressure sensors 1 and 2. The coherence threshold based on $n_{d}=250$ is shown by the broken horizontal line at $\hat{\gamma}_{x_{n} y_{n}}^{2}\left(f, P_{I}=0.95, n_{d}=250\right) \approx 0.012$. The coherence threshold based on $n_{s}$ $=500$ is shown by the unbroken horizontal line at $\hat{\gamma}_{x_{n} y_{n}}^{2}\left(f, P_{I}=0.95\right.$, $\left.n_{d}=500\right) \approx 0.006$

(lighter line), which produces a rougher density estimate. A normal distribution is shown as a dashed curve. A onedimensional scatter plot is shown at the bottom of each graph, using a vertical bar as the plotting symbol, with the horizontal location given by coherence. On this plot, vertical lines indicate statistical estimates of the upper $95 \%$ threshold coherence confidence limit of the un-aligned coherence. Using $n_{d}=250$ produces a vertical line at $w(0.012) \approx 0.109$ since $\hat{\gamma}_{x_{n} y_{n}}^{2}(250)=0.012$ while using $n_{s}=500$ produces a vertical line at $w(0.006) \approx 0.077$ since $\hat{\gamma}_{x_{n} y_{n}}^{2}(500)=0.006$. Again, for this turbofan engine data case, the vertical based on $n_{d}=250$ provides a better coherence threshold.

To investigate the frequency dependence of the engine coherence data with coherence greater than a threshold coherence value of $\hat{\gamma}_{x_{n} y_{n}}^{2}\left(n_{s}=500\right)=0.006$, these points were culled from a number of un-aligned coherence plots for a range of operating conditions and are shown in a scatter plot in Fig. 6. In addition, the lower horizontal statistical coherence threshold line $\hat{\gamma}_{x_{n} y_{n}}^{2}\left(n_{s}\right)=\hat{\gamma}_{x_{n} y_{n}}^{2}\left(f, P_{I}=0.95, n_{s}=500\right)$ $\approx 0.006$ and the higher horizontal statistical coherence threshold line $\hat{\gamma}_{x_{n} y_{n}}^{2}\left(n_{d}\right)=\hat{\gamma}_{x_{n} y_{n}}^{2}\left(f, P_{I}=0.95, n_{s}=250\right)$ $\approx 0.012$ calculated from Eq. (7) are shown. This plot clearly indicates sinusoids-in-noise between 400 and $500 \mathrm{~Hz}$. In addition, the broken horizontal line with a coherence threshold value $\hat{\gamma}_{x_{n} y_{n}}^{2}\left(n_{d}=250\right)=0.012$ is clearly a better choice of a coherence threshold. With this decision, most of the scattered culled points are interpreted as being due to statistical random fluctuations and not tones.

Comparing the engine test un-aligned results shown in Fig. 3-5 with the simulated noise un-aligned results shown in Figs. 2, it is obvious that using the value of the number of disjoint (independent) segments, $n_{d}$, provides a better estimate of the coherence threshold for the engine data. However, using the number of segments with overlap, $n_{s}$, provides a good threshold estimate for the data simulated using a Gaussian distribution of values. In the next few sections, turbofan engine core noise measurements using the three signal coherence technique and the coherent output power calculation are examined using these statistical coherence threshold values.

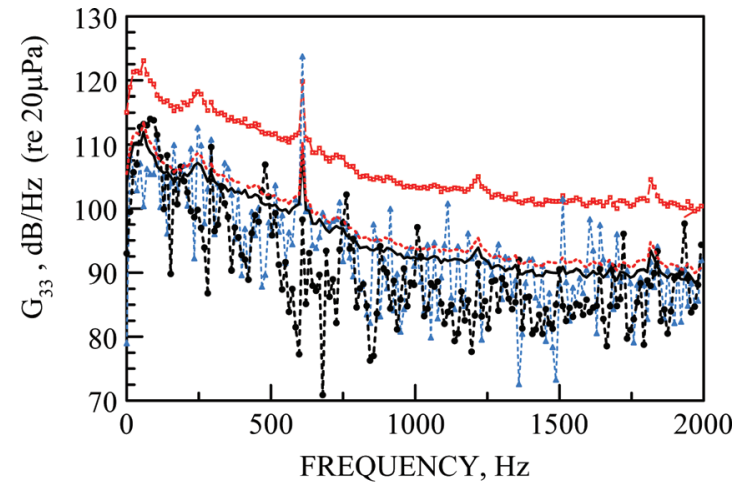

$$
\begin{aligned}
& \cdots-G_{33} \\
& \cdots-G_{33}=\left|G_{13}(D=0)\right|\left|G_{23}(D=0)\right| /\left|G_{12}(D=6323)\right| \text { unaligned } \\
& \left.\cdots \cdots G_{33}=\left|G_{13}(D=6839)\right|\left|G_{23}(D=6134)\right| /\left|G_{12}(D=0)\right|\right) \text { aligned } \\
& \cdots \cdots G_{33} \gamma_{n n}\left(n_{d}=250\right) \\
& -G_{33} \gamma_{n n}\left(n_{d}=500\right)
\end{aligned}
$$

FIG. 7. (Color online) Three signal coherence technique using one far-field microphone and two combustor pressure sensors. The dashed line is based on an estimate of the upper $95 \%$ confidence limit of the un-aligned coherence threshold using $n_{d}=250\left(\hat{\gamma}_{x_{n} y_{n}}^{2}\left(f, P_{I}=0.95, n_{d}=250\right) \approx 0.012\right)$. The heavy line is based on an estimate of the upper $95 \%$ confidence limit of the un-aligned coherence threshold using $n_{s}=500\left(\hat{\gamma}_{x_{n} y_{n}}^{2}\left(f, P_{i}=0.95\right.\right.$, $\left.\left.n_{s}=500\right) \approx 0.006\right)$.

\section{B. Three signal coherence technique}

The three signal coherence technique $e^{21,22}$ is applied to noise from a P\&W4098 turbofan engine using the aligned and un-aligned coherence functions. Typical results of applying the three signal and un-aligned coherence technique are shown in Figs. 7 and 8. Results calculated using signals from one far-field microphone and two combustor pressure sensors are shown in Fig. 7. Results calculated using signals from three far-field microphones are shown in Fig. 8.

\section{Three signal coherence calculated using one far-field microphone and two combustor pressure sensors}

The example shown is for $1622 \mathrm{rpm}$ (N1 Corr.). The three signal aligned and un-aligned coherence calculated using the microphone at $100^{\circ}$ (sensor 3) and the two combustor pressure sensors (sensors 1 and 2) are used to show the presence of combustion noise in the region from 0 to 200 $\mathrm{Hz}$ in Fig. 7. The results are shown so that they can be compared to auto-spectrum, $\hat{G}_{33}$, shown as the top curve composed of squares in Fig. 7. The aligned three signal spectrum, $\left.\hat{G}(f)_{v_{3} v_{3}}\right|_{\text {aligned }}$, shown by black circles is given by

$$
\left.\hat{G}(f)_{v_{3} v_{3}}\right|_{\text {aligned }}=\left.\hat{G}(f)_{y_{3} y_{3}} \frac{\hat{\gamma}(f)_{13} \hat{\gamma}(f)_{23}}{\hat{\gamma}(f)_{12}}\right|_{\text {aligned }} \text {. }
$$

The un-aligned three signal spectrum, $\left.\hat{G}(f)_{v_{3} v_{3}}\right|_{\text {unaligned }}$, shown by triangles is given by

$$
\hat{G}(f)_{v_{3} v_{3}} \mid \text { unaligned }=\left.\hat{G}(f)_{y_{3} y_{3}} \frac{\hat{\gamma}(f)_{13} \hat{\gamma}(f)_{23}}{\hat{\gamma}(f)_{12}}\right|_{\text {unaligned }} \text {. }
$$




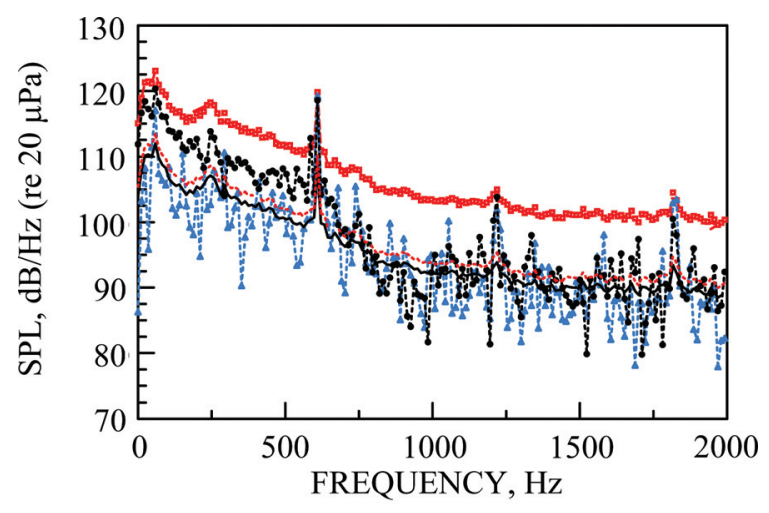

$\cdots \mathrm{G}_{33}$
$\cdots \mathrm{G}_{33} \gamma_{34} \gamma_{35} / \gamma_{45}$ unaligned
$\cdots \cdots \mathrm{G}_{33} \gamma_{34} \gamma_{35} / \gamma_{45}$ aligned
$\cdots \cdots \mathrm{G}_{33} \gamma_{\mathrm{nn}}\left(\mathrm{n}_{\mathrm{d}}=250\right)$
$-\mathrm{G}_{33} \gamma_{\mathrm{nn}}\left(\mathrm{n}_{\mathrm{d}}=500\right)$

FIG. 8. (Color online) Three signal coherence technique using three farfield microphones. The dashed line is based on an estimate of the upper 95\% confidence limit of the un-aligned coherence threshold using $n_{d}=250$ $\left(\hat{\gamma}_{x_{n} y_{n}}^{2}\left(f, P_{I}=0.95, n_{d}=250\right) \approx 0.012\right)$. The heavy line is based on an estimate of the upper $95 \%$ confidence limit of the un-aligned coherence threshold using $n_{s}=500\left(\hat{\gamma}_{x_{n} y_{n}}^{2}\left(f, P_{i}=0.95, n_{s}=500\right) \approx 0.006\right)$.

The upper and lower coherence threshold spectra are also shown. The upper threshold $\hat{G}_{33} \hat{\gamma}_{x_{n} y_{n}}^{2}\left(n_{d}\right)$ is shown by the dashed curve. The lower threshold $\hat{G}_{33} \hat{\gamma}_{n_{n} y_{n}}^{2}\left(n_{s}\right)$ is shown by the black curve. The un-aligned three signal spectrum $\hat{G}(f)_{v_{3} v_{3}}$ unaligned is below the coherence threshold at most frequencies except for the presence of tones. The aligned three signal spectrum $\left.\hat{G}(f)_{v_{3} v_{3}}\right|_{\text {aligned }}$ is above the coherence threshold curves in the region $0-200 \mathrm{~Hz}$. This shows the presence of combustion noise. Results from a study of noise from a smaller Honeywell TECH977 dual-spool turbofan engine by Miles ${ }^{27-29}$ suggest this noise is due to an indirect combustion noise source.

The translation procedure will be discussed next. The number of translation points, $D$, used for each cross-spectrum so that the signals of interest would be in proper alignment is given in the legend. To calculate the aligned $\hat{G}_{33}$ spectrum, a delay of 6839 time steps was used in calculating the aligned cross-spectrum $\hat{G}_{13}$ to remove the time delay caused by the signal traveling between and microphone 3 and combustor sensor 1. A delay of $D=6134$ time steps was used in calculating the $\hat{G}_{23}$ aligned cross-spectrum. The delay times were selected by maximizing the coherence and making the plot of the cross spectrum phase angle as a function of frequency flat. The $\hat{G}_{12}$ cross-spectrum was used as measured. To calculate the un-aligned $\hat{G}_{33}$ spectrum a delay of 6323 time steps was used in calculating the cross spectrum $\hat{G}_{12}$, and the $\hat{G}_{13}$ and $\hat{G}_{23}$ cross-spectrum were used as measured. The $D=6839$ and $D=6134$ time steps are in the range of the travel time from the turbofan to the microphones used. However, some attempt was made to find time intervals that maximized the coherence in the 0 to $200 \mathrm{~Hz}$ frequency band and those are used here.

Tone noise appears in both the aligned and un-aligned poser spectrum. The tone at one BPF (first blade passing fre- quency) is especially noticeable. However, it is best shown in the un-aligned power spectrum where the broad band noise does not appear. Note that one can view tones approximately $10 \mathrm{~dB}$ below the measured auto-spectrum in the three signal coherent aligned and un-aligned power spectrum. In the un-aligned three signal spectrum many tones previously unseen are now exposed to sight since the coherent broadband noise has been removed. The aligned and un-aligned procedure solves the sinusoids-in-noise and hidden periodicity problem for turbofan engine noise.

\section{Three signal coherence calculated using far-field microphones}

Three signal aligned and un-aligned power spectra calculated using far-field microphones at $100^{\circ}$ (sensor 3), $110^{\circ}$ (sensor 4), and $120^{\circ}$ (sensor 5) are shown in Fig. 8. As with Fig. 7, the results are shown so that they can be compared to auto-spectrum, $\hat{G}_{33}$, shown as the top curve composed of squares in Fig. 8. The plot clearly shows the tonal component due to the core and fan in the far-field noise spectrum, $\left.\hat{G}_{u_{3} u_{3}}\right|_{\text {unaligned }}$, created using the un-aligned coherence (light diamonds) where

$$
\hat{G}(f)_{u_{3} u_{3}} \mid \text { unaligned }=\left.\hat{G}(f)_{y_{3} y_{3}} \frac{\hat{\gamma}(f)_{34} \hat{\gamma}(f)_{35}}{\hat{\gamma}(f)_{45}}\right|_{\text {unaligned }} \text {. }
$$

Above this spectrum is a spectrum $\left.\hat{G}_{u_{3} u_{3}}\right|_{\text {aligned }}$ created using the aligned coherence (dark dots) where

$$
\left.\hat{G}(f)_{u_{3} u_{3}}\right|_{\text {aligned }}=\left.\hat{G}(f)_{y_{3} y_{3}} \frac{\hat{\gamma}(f)_{34} \hat{\gamma}(f)_{35}}{\hat{\gamma}(f)_{45}}\right|_{\text {aligned }} .
$$

The upper and lower coherence threshold spectra are also shown. The upper threshold $\hat{G}_{33} \hat{\gamma}_{x_{n} y_{n}}^{2}\left(n_{d}\right)$ is shown by the dashed curve. The lower threshold $\hat{G}_{33} \hat{\gamma}_{x_{n} y_{n}}^{2}\left(n_{s}\right)$ is shown by the black curve. The un-aligned three signal spectrum $\left.\hat{G}(f)_{u_{3} u_{3}}\right|_{\text {unaligned }}$ is below the coherence threshold at most frequencies except for the presence of tones. The aligned three signal spectrum $\left.\hat{G}(f)_{v_{3} v_{3}}\right|_{\text {aligned }}$ is far above the coherence threshold curves in the region $0-500 \mathrm{~Hz}$. This shows the presence of a strong low frequency coherent noise source.

The aligned coherence values were used as measured. The un-aligned coherence values were found by delaying one signal 6323 time units with respect to the other. The jet noise is missing in the three signal aligned and un-aligned power spectra to the extent that the microphones are far enough apart that the jet noise signals they receive are independent in this frequency range. This feature of jet noise is exploited by the three signal coherence technique using three far-field microphones. The aligned and un-aligned spectra from 0 to $500 \mathrm{~Hz}$ are well separated showing the presence of a coherent noise source.

However, this noise source could not be attributed to core noise. Furthermore, interpreting the coherence measurement used to obtain the coherent output power was made difficult due to the presence of an external extraneous low frequency noise source as discussed by Miles ${ }^{5}$ 
which reduces the measured coherence and required the calculation of a coherence threshold. This external extraneous low frequency noise source is the major contributor to the three far-field microphone three signal coherence shown in Fig. 8.

Figure 8 clearly shows the broadband noise spectrum and the tonal component where it dominates. Above this is a broadband spectrum interrupted in places by tonal noise. The 1BPF, 2BPF, and 3BPF tones are clearly observable along with many other tones in the three signal aligned and un-aligned power spectra. Note again, that one can view tones approximately $10 \mathrm{~dB}$ below the measured auto-spectrum in the three signal coherent aligned and un-aligned power spectrum. Similar behavior appears at other turbofan engine speeds.

\section{Coherent output power}

Two typical coherent output power ${ }^{15}$ calculations using combustor pressure sensor 1 and 2 with the $120^{\circ}$ far-field microphone (signal source 5) are presented in Fig. 9 for a test condition of $1622 \mathrm{rpm}$ (N1 Corr.). These results are shown so that they can be compared to auto-spectrum, $G_{55}$, shown as the top curve composed of squares in Fig. 9. To obtain the aligned coherent output power using combustor pressure sensor 2 and signal source 5 shown by the open diamonds, a time delay of $0.116875 \mathrm{~s}$ was used. To obtain the aligned coherent output power for combustor pressure sensor 1 and signal source 5 shown by the open circles, a time delay of $0.128083 \mathrm{~s}$ was used. The plane wave combustion noise is apparent between 0 and

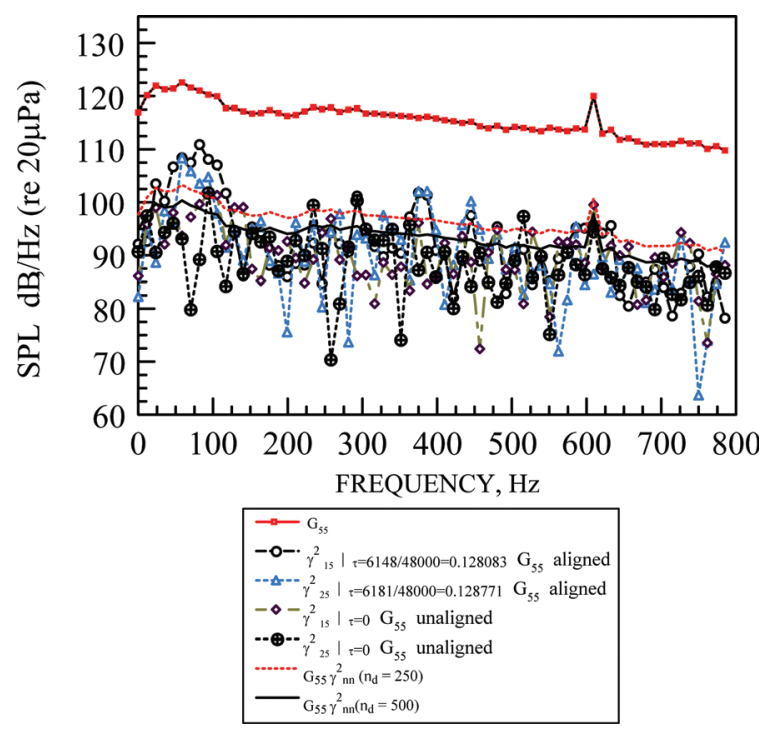

FIG. 9. (Color online) Total sound power, aligned and un-aligned coherent output power calculation for a test condition of $1622 \mathrm{rpm}$ (N1 CORR), using signal 1 (pressure sensor 1 at $127^{\circ}$ clockwise from top dead center viewed from rear) and 2 (pressure sensor 2 at $337^{\circ}$ clockwise from top dead center viewed from rear) with signal $5\left(150\right.$ foot microphone at $\left.120^{\circ}\right)$. The light dashed line is based on an estimate of the upper $95 \%$ confidence limit of the un-aligned coherence threshold using $n_{d}=250\left(\hat{\gamma}_{x_{y}}^{2}\left(f, P_{I}=0.95\right.\right.$, $\left.n_{d}=250\right) \approx 0.012$ ). The heavy line is based on an estimate of the upper $95 \%$ confidence limit of the un-aligned coherence threshold using $n_{s}=500\left(\hat{\gamma}_{x_{n} y_{n}}^{2}\left(f, P_{i}=0.95, n_{s}=500\right) \approx 0.006\right)$.
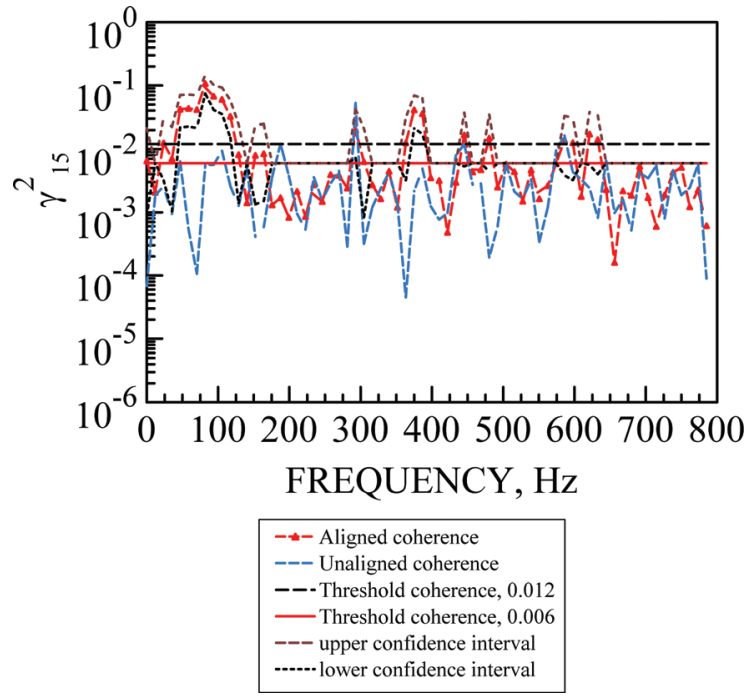

FIG. 10. (Color online) Aligned coherence, $\hat{\gamma}_{x y}^{2}(f)$, and un-aligned coherence, $\left|\hat{\gamma}_{x, y}^{2}(f)\right|$, calculation for a test condition at $1622 \mathrm{rpm}$ (N1 CORR) using signal 1 (pressure sensor 1 at $127^{\circ}$ clockwise from top dead center viewed from rear) and 5 (150 foot microphone at $\left.120^{\circ}\right)$. The broken horizontal line at $\hat{\gamma}_{x_{n} y_{n}}^{2}\left(f, P_{I}=0.95, n_{d}=250\right) \approx 0.012$ represents an estimate of the upper $95 \%$ confidence limit of the un-aligned coherence for $n_{d}=250$. The unbroken horizontal line at $\hat{\gamma}_{x_{n} y_{n}}^{2}\left(f, P_{i}=0.95, n_{s}=500\right) \approx 0.006$ represents an estimate of the upper $95 \%$ confidence threshold limit of the unaligned coherence for $n_{s}=500$.

$200 \mathrm{~Hz}$ in both aligned coherent output power spectra $\left.\hat{\gamma}_{15}^{2}(f) \hat{G}_{55}(f)\right|_{\text {aligned }}$ and $\left.\hat{\gamma}_{25}^{2}(f) \hat{G}_{55}(f)\right|_{\text {aligned }}$. Both spectra are clearly above their corresponding un-aligned coherent output power spectra calculated as $\left.\hat{\gamma}_{15}^{2}\right|_{\text {unaligned }} G_{55}(f)$ and $\hat{\gamma}_{25}^{2} \mid$ unaligned $G_{55}(f)$. The un-aligned coherent output power spectra were obtained using the as measured signals with no time delay.

Statistical threshold coherent output power curves given by $\hat{\gamma}_{x_{n} y_{n}}^{2}\left(n_{d}=250\right) \hat{G}_{55}(f)$ and $\hat{\gamma}_{x_{n} y_{n}}^{2}\left(n_{s}=250\right) \hat{G}_{55}(f)$ are also shown in Fig. 9. The measured aligned coherent output power spectra near $100 \mathrm{~Hz} \quad\left(\left.\hat{\gamma}_{15}^{2}(f) \hat{G}_{55}(f)\right|_{\text {aligned }}\right.$ and $\hat{\gamma}_{25}^{2}(f) G_{55}$ $\left.\left.(f)\right|_{\text {aligned }}\right)$ are clearly above the statistical coherence threshold output power curves. These results suggest the presence of indirect combustion noise at low frequencies due to interaction of hot spots with the turbine since they are similar to the results discussed by Miles ${ }^{27-29}$ for a smaller turbofan engine.

The results also suggest that combustion noise due to higher combustion duct modes is present near 300, 400, and $600 \mathrm{~Hz}$. Note that the noise floor is about $20 \mathrm{~dB}$ below the measured auto-spectrum.

The corresponding aligned and un-aligned coherence between pressure sensor 1 and the $120^{\circ}$ far-field microphone (sensor 5) are shown in Fig. 10. The upper and lower coherence confidence interval bands for the aligned coherence are also shown. In addition, the lower horizontal statistical coherence threshold line $\hat{\gamma}_{x_{n} y_{n}}^{2}\left(n_{s}\right)=\hat{\gamma}_{x_{n} y_{n}}^{2}\left(f, P_{I}=0.95, n_{s}=500\right)$ $\approx 0.006$ and the higher horizontal statistical coherence threshold line $\hat{\gamma}_{x_{n} y_{n}}^{2}\left(n_{d}\right)=\hat{\gamma}_{x_{n} y_{n}}^{2}\left(f, P_{I}=0.95, n_{s}=250\right) \approx 0.012$ calculated from Eq. (7) are shown. The plane wave combustion noise is apparent between 0 and $200 \mathrm{~Hz}$ in the aligned coherence function. These are the coherence functions used to create Fig. 9. 
TABLE II. Time series estimation parameters. $[r=48000$ samples/s, $N P=$ number of points in FFT $=4096$, $\left.P=0.95, B_{e}=r / N P=11.71875 \mathrm{~Hz}, n_{d}=B_{e} T_{\text {total }}\right\}=$ number of disjoint (independent) segments]

\begin{tabular}{|c|c|c|c|c|c|}
\hline $\begin{array}{l}\text { Total record } \\
\text { length, } T_{\text {total }} \text { s }\end{array}$ & Overlap & $n$ & $\begin{array}{c}\hat{\gamma}_{x_{n} y_{n}}^{2}= \\
1-\left(1-P_{I}\right)^{1 /(n-1)}\end{array}$ & $\begin{array}{l}\text { 3-signal noise } \\
\text { floor, dB }\end{array}$ & $\begin{array}{c}\text { Coherent output } \\
\text { power noise floor, } \mathrm{dB}\end{array}$ \\
\hline 20 & 0 & 234 & 0.0127749 & -9.468 & -18.936 \\
\hline 20 & $50 \%$ & $\approx 468$ & 0.00639431 & -10.971 & -21.9421 \\
\hline 120 & 0 & 1406 & 0.00221992 & -13.3582 & -26.7164 \\
\hline 120 & $50 \%$ & $\approx 2812$ & 0.00106515 & -14.8629 & -28.7259 \\
\hline 300 & 0 & 3515 & 0.00085215 & -15.3474 & -30.6948 \\
\hline 300 & $50 \%$ & $\approx 7030$ & 0.000426105 & -16.8524 & -33.7048 \\
\hline
\end{tabular}

\section{DISCUSSION}

Table II shows that using longer record lengths, $T_{\text {total }}$, of $2 \mathrm{~min}$ or $5 \mathrm{~min}$ provide a significantly lower noise floor. Note that the coherent power noise floor is always less than the coherence noise floor based on the three signal method being reduced by the square of the coherence.

Two standard statistical tests were applied to the Fisher z-transformed un-aligned coherence results obtained analyzing simulated time histories and measured turbofan engine time histories to determine if they had a normal distribution. A norm Q-Q comparison plot showed the simulated time history data that produced a more normal distribution than the turbofan engine data which had outliers, i.e., points that are numerically distant from the rest of the data. The second test was to use a histogram plot. This also showed the presence of outliers in the measurements made using the turbofan engine data. The threshold statistical coherence based on the number of independent samples provided a conservative value. This value appeared appropriate for the turbofan engine data. However, it appeared that a smaller value based on the actual number of overlapped samples would be more appropriate for the simulated data.

Finding hidden periodicities in signals with additive noise is a signal processing problem with many solutions. ${ }^{13}$ However, the aligned and un-aligned coherence method might be useful in cases where the problem involves long time histories having an unknown number of multiple periodic signals and a bad signal to noise ratio. The un-aligned coherence procedure has very low computational cost compared to the other signal processing methods. The method might be useful in performing blind test comparisons to validate and compare these other methods performance on non synthetic or simulated time histories. Looking at the literature such a method does not appear available.

The three signal coherence technique calculations using one far-field microphone ant two combustor pressure sensors shown in Fig. 7 and the total sound power calculations shown in Fig. 9 suggest the presence of indirect combustion noise at low frequencies due to the interaction of hot spots with the turbine and are similar to the results discussed by Miles ${ }^{27-29}$ obtained using a smaller Honeywell TECH977 turbofan engine. The results obtained in the study of the smaller turbofan engine were identified using the convection time delay associated with the flow velocity of the entropy in the combustor. For this smaller engine, coherence values between the combustor sensor and the far-field microphones were much larger and the cross-spectrum phase angle provided information on the convection time delay which allowed the identification of indirect combustion noise.

\section{CONCLUDING REMARKS}

In calculating autospectra and cross-spectra using the averaged periodogram method, the records used are frequently overlapped to reduce the variance. In calculating the coherence threshold using a statistical method one may use either the number of independent samples or a larger number corresponding to the number of overlapped records used to create the average. This paper indicates how applying the Fisher z-transform to the un-aligned coherence can aid in making the proper selection of samples and produce a reasonable coherence threshold. The paper shows that using the number of independent samples produces a conservative statistical coherence threshold useful for the turbofan engine data studied herein.

For the simulated time histories based on Gaussian statistics used herein, the statistical coherence threshold based on a larger number of segments corresponding to the number of overlapped records had a lower value. This value is shown to be more appropriate by use of the aligned and un-aligned coherence and the Fisher z-transform.

A method has been developed to help identify the coherent combustion noise source using far-field microphone data contaminated with low frequency noise from other sources. The method uses an aligned and un-aligned coherence technique to determine the significance of the coherence and to establish a threshold level for the coherence. This method is compared with a statistics based method that can be used to calculate the coherence threshold. When used with the classical three signal coherence power technique and the coherent power method these methods provides information on coherent combustion noise in the presence of an external extraneous low frequency coherent noise source. In addition, the un-aligned coherence procedure provides a method to separate tonal information from random process information. The procedure at the same time as it calculates a coherence threshold also reveals periodicities, spectral lines, and undamped sinusoids hidden or concealed by broadband turbofan engine noise. Comparison of these results with those obtained by another turbofan engine combustion noise 
source detection study using a smaller engine suggest the low frequency combustion noise observed is due to an indirect combustion noise source. It is expected that this method will improve turbofan source diagnostic tests.

\section{ACKNOWLEDGMENTS}

This work was supported by the NASA Fundamental Aeronautics Subsonic Fixed Wing program.

${ }^{1}$ P. D. Welch, "The use of fast Fourier transform for the estimation of power spectra: A method based on time averaging over short, modified periodograms," IEEE Trans. Audio Electroacoust. 15(2), 70-73 (1967).

${ }^{2}$ J. V. Canday, Signal Processing. The Modern Approach (McGraw-Hill, New York, 1988), pp. 149-177, 178-353.

${ }^{3}$ S. D. Stearns and R. A. David, Signal Processing Algorithms Using FORTRAN and C (Prentice-Hall, Englewood Cliffs, NJ, 1993), Chap. 4, pp. 291-295.

${ }^{4}$ D. G. Manolakis, V. K. Ingle, and S. M. Kogon, Statistical and Adaptive Signal Processing (Artech House, Boston, MA, 2005), Chaps. 5, 9.

${ }^{5}$ J. H. Miles, "Separating turbofan engine noise sources using auto and cross spectra from four microphones," AIAA J. 46(1), 61-74 (2008).

${ }^{6}$ G. C. Carter, "Receiver operating characteristics for a linearly thresholded coherence estimation detector," IEEE Trans. Acoust., Speech, Signal Process. 25, 90-92 (1977).

${ }^{7}$ G. C. Carter, "Coherence and time delay estimation," Proc. IEEE 75(2), 236-255 (1987).

${ }^{8}$ D. M. Halliday, J. R. Rosenberg, A. M. Amjad, P. Breeze, B. A. Conway, and S. F. Farmer, "A framework for the analysis of mixed time series/ point process data-theory and application to the study of physiological tremor, single motor unit discharges and electromyograms," Prog. Biophys. Mol. Biol. 64(2-3), 237-278 (1995).

${ }^{9}$ D. R. Brillinger, Time Series: Data Analysis and Theory -Expanded Edition (Holden-Day, San Francisco, CA, 1981), pp. 266, 294, 314-317.

${ }^{10}$ R. B. Blackman and J. W. Tukey, The Measurement of Power Spectra (Dover Publications, New York, 1958), pp. 1-190.

${ }^{11} \mathrm{~J}$. H. Miles, "Aligned and unaligned coherence: A new diagnostic tool, AIAA Paper No. 2006-0010," Technical Report NASA/TM2006-214112, NASA, Cleveland, OH (2006).

${ }^{12}$ L. Faes, G. D. Pinna, A. Porta, R. Maestri, and G. Nollo, "Surrogate data analysis for assessing the significance of the coherence function," IEEE Trans. Biomed. Eng. 51(7), 1156-1166 (2004).

${ }^{13}$ C. L. Byrne, Signal Processing: A Mathematical Approach (A. K. Peters, Wellesley, MA, 2005), Chaps. 33-43.

${ }^{14}$ B. G. Quinn and E. J. Hannan, The Estimation and Tracking of Frequency (Cambridge University Press, United Kingdom, 2001), pp. 1-266.

${ }^{15}$ J. S. Bendat and A. G. Piersol, Engineering Applications of Correlation and Spectral Analysis (Wiley, New York, 1980), pp. 83-84, 272.

${ }^{16}$ G. M. Jenkins and D. G. Watts, Spectral Analysis and Its Applications (Holden-Day, San Francisco, CA 1969), pp. 352, 356-359, 399-400.

${ }^{17}$ M. Priestley, Spectral Analysis and Time Series (Elsevier Academic Press, San Diego, CA, 1981), p. 712.

${ }^{18}$ G. C. Carter, C. H. Knappy and A. H. Nuttall, "Estimation of the magnitude-squared coherence function via overlapped fast Fourier transform processing," IEEE Trans. Audio, Electroacoust. 21(4), 337-344 (1973).

${ }^{19}$ J. Gross, P. A. Tass, S. Salenius, R. Hari, H.-J. Freund, and A. Schnitzler, "Cortico-muscular synchronization during isometric muscle contraction in humans as revealed by magnetoencephalography," J. Physiol. 527(3), 623-631 (2000).

${ }^{20} \mathrm{R}$. A. Fisher, "The general sampling distribution of the multiple correlation coefficient," Proc. R. Soc. Lond. A 121, 654-673 (1928).

${ }^{21}$ J. Y. Chung, M. J. Crocker, and J. F. Hamilton, "Measurement of frequency response and the multiple coherence function of the noise-generation system of a diesel engine," J. Acoust. Soc. Am. 58(3), 636-642 (1975).

${ }^{22}$ J. Y. Chung, "Rejection of flow noise using a coherence function method," J. Acoust. Soc. Am. 62(2), 388-395 (1977).
${ }^{23}$ J. H. Miles, "Validating coherence measurements using aligned and unaligned coherence functions, AIAA Paper No. 2006-1389," Technical Report NASA/TM2006-214113, NASA, Cleveland, OH (2006).

${ }^{24}$ A. M. Karchmer, "Acoustic modal analysis of a full scale annular combustor, AIAA Paper No. 1983-0760," Technical Report NASA TM-83334, NASA, Clevelamd, OH(1983).

${ }^{25}$ J. Tyler and T. Sofrin, "Axial flow compressor noise studies," Trans. Soc. Automot. Eng. 70, 309-332 (1962).

${ }^{26} \mathrm{~J}$. H. Miles, "Restricted modal analysis applied to internal annular combustor autospectra and cross-spectra measurements," AIAA J. 45(5), 988999 (2007).

${ }^{27} \mathrm{~J}$. H. Miles, "Time delay analysis of turbofan engine direct and indirect combustion noise sources," J. Propul. Power 25(1), 218-227 (2009).

${ }^{28} \mathrm{~J}$. Miles, "Core noise diagnostics of turbofan engine noise using correlation and coherence functions," J. Propul. Power 26(2), 303-316 (2010).

${ }^{29}$ J. Miles, "Separating direct and indirect turbofan engine combustion noise using the correlation function," J. Propul. Power 26(5), 1144-1152 (2010).

${ }^{30}$ S. Becker, C. Hahn, M. Kaltenbacher, and R. Lerch, "Flow-induced sound of wall-mounted cylinders with different geometries," AIAA J. 46(9), 2265-2281(2008).

${ }^{31}$ J. M. Mendoza, D. K. Nance, and K. K. Ahuja, "Source separation from multiple microphone measurements in the far field of a full scale aero engine, AIAA Paper No. 2008-2809.

${ }^{32} \mathrm{D}$. S. Weir, "Engine validation of noise \& emission reduction technology phase 1," Technical Report, No. NASA/CR-2008-215225, NASA, Cleveland, OH, Honeywell Technical Report No. 2113843A, Honeywell, Phoenix, Arizona (2008).

${ }^{33} \mathrm{R}$. Bortel and P. Sovka, "Approximation of statistical distribution of magnitude squared coherence estimated with segment overlapping," Signal Process. 87(5) 1100-1117 (2007).

${ }^{34}$ A. Piersol, "Time delay estimation using phase data," IEEE Trans. Acoust., Speech, Signal Process. 29(3), 471-477 (1981).

${ }^{35}$ J. S. Bendat and A. G. Piersol, Random Data: Analysis and Measurement Procedures (Wiley, New York, 1971), pp. 193-194.

${ }^{36}$ S.-Y. Wang, X. Liu, J. Yianni, R. C. Miall, T. Z. Aziz, and J. F. Stein, "Optimizing coherence estimation to assess the functional correlation of tremor-related activity between the subthalamic nucleus and the forearm muscles," J. Neurosci. Methods 136(2), 197-205 (2004).

${ }^{37} \mathrm{~S}$. Y. Wang and M. Tang, "Exact confidence interval for magnitude-squared coherence estimates," IEEE Signal Process. Lett. 11(3), 326-329 (2004).

${ }^{38} \mathrm{~N}$. R. Goodman, "On the joint estimation of the spectra, cospectrum, and quadrature spectrum of a two-dimensional stationary Gaussian process," Technical Report Scientific paper No. 10, Engineering Statistics Laboratory of New York University (1957), AD-134919 (Also Ph.D. dissertation, Princeton University).

${ }^{39}$ J. J. Gosselin, "Comparative study of two-sensor (magnitude-squared coherence) and single-sensor (square-law) receiver operating characteristics," in Proceedings of the IEEE International Conference on Acoustics, Speech, and Signal Processing, Hartford, CT (1977) pp. 311-314.

${ }^{40} \mathrm{~A}$. H. Nuttall, "Invariance of distribution of coherence estimate to secondchannel statistics," IEEE Trans. Acoust., Speech, Signal Process. 29(1), 120-122 (1981)

${ }^{41}$ A. Nuttall and G. Carter, "An approximation to the cumulative distribution function of the magnitude-squared coherence estimate," IEEE Trans. Acoust., Speech, Signal Process. 29(4), 932-934 (1981).

${ }^{42} \mathrm{~J}$. Fox, An R and S-Plus Companion to Applied Regression (Sage Publications, Thousand Oaks, CA, 2002), pp. 85-91.

${ }^{43}$ M. R. Foster and N. J. Guinzy, "The coefficient of coherence: Its estimation and use in geophysical data processing," Geophysics 32(4), 602-616 (1967).

${ }^{44} \mathrm{~V}$. A. Benignus, "Estimation of the coherence spectrum and its confidence interval using the fast Fourier transform," IEEE Trans. Audio Electroacoust 17(2), 145-150 (1969).

${ }^{45} \mathrm{~J}$. W. Tukey, "An introduction to the calculations of numerical spectrum analysis," in Spectral Analysis of Time Series edited by B. Harris (Wiley, New York, 1967) pp. 25-46, 32. 